\title{
FROM BLUE STAR-FORMING TO RED PASSIVE: GALAXIES IN TRANSITION IN DIFFERENT ENVIRONMENTS
}

\author{
Benedetta Vulcani ${ }^{1}$, Bianca M. Poggianti ${ }^{2}$, Jacopo Fritz $^{3,4}$, Giovanni Fasano ${ }^{2}$, \\ Alessia Moretti ${ }^{2,5}$, Rosa Calvi ${ }^{5}$, and Angela Paccagnella ${ }^{5}$ \\ ${ }^{1}$ Kavli Institute for the Physics and Mathematics of the Universe (WPI), Todai Institutes for Advanced Study, the University of Tokyo, Kashiwa 277-8582, Japan \\ ${ }^{2}$ INAF-Astronomical Observatory of Padova, I-35122 Padova, Italy \\ ${ }^{3}$ Sterrenkundig Observatorium Vakgroep Fysica en Sterrenkunde Universiteit Gent, Krijgslaan 281, S9 B-9000 Gent, Belgium \\ ${ }^{4}$ Centro de Radioastronomía y Astrofísica, CRyA, UNAM, Campus Morelia, A.P. 3-72, C.P. 58089 Michoacán, Mexico \\ ${ }^{5}$ Dipartimento di Fisica e Astronomia, Universitá degli Studi di Padova, vicolo Osservatorio 2, I-35122 Padova, Italy \\ Received 2014 July 23; accepted 2014 October 23; published 2014 December 19
}

\begin{abstract}
Exploiting a mass-complete $\left(M_{*}>10^{10.25} M_{\odot}\right)$ sample at $0.03<z<0.11$ drawn from the Padova Millennium Galaxy Group Catalog, we use the $(U-B)_{r f}$ color and morphologies to characterize galaxies, in particular those that show signs of an ongoing or recent transformation of their star-formation activity and/or morphology: green galaxies, red passive late types, and blue star-forming early types. Color fractions depend on mass and only for $M_{*}<10^{10.7} M_{\odot}$ on environment. The incidence of red galaxies increases with increasing mass, and, for $M_{*}<10^{10.7} M_{\odot}$, decreases toward the group outskirts and in binary and single galaxies. The relative abundance of green and blue galaxies is independent of environment and increases monotonically with galaxy mass. We also inspect galaxy structural parameters, star-formation properties, histories, and ages and propose an evolutionary scenario for the different subpopulations. Color transformations are due to a reduction and suppression of the starformation rate in both bulges and disks that does not noticeably affect galaxy structure. Morphological transitions are linked to an enhanced bulge-to-disk ratio that is due to the removal of the disk, not to an increase of the bulge. Our modeling suggests that green colors might be due to star-formation histories declining with long timescales, as an alternative scenario to the classical "quenching" processes. Our results suggest that galaxy transformations in star-formation activity and morphology depend neither on the environment nor on being a satellite or the most massive galaxy of a halo. The only environmental dependence we find is the higher fast quenching efficiency in groups giving origin to poststarburst signatures.
\end{abstract}

Key words: galaxies: elliptical and lenticular, $\mathrm{cD}$ - galaxies: fundamental parameters - galaxies: groups: general galaxies: spiral - galaxies: star formation - galaxies: structure

\section{INTRODUCTION}

Galaxy color and structure are key observables in extragalactic astronomy for understanding the formation and evolution of galaxies, and they are the consequence of all physical processes at work.

The local population of galaxies consists roughly of two types, and their frequency correlates with the environment: red galaxies, which on the whole are characterized by larger stellar masses and bulge-dominated morphologies and are predominant in dense regions, and blue galaxies, with a disk-dominated morphology, which are preferentially found in low-density regions (Blanton et al. 2003; Kauffmann et al. 2003, 2004; Baldry et al. 2004; Balogh et al. 2004; Brinchmann et al. 2004). Because only relatively small amounts of ongoing star formation make a galaxy appear blue, the color bimodality basically reflects star-formation quenching: in general, red galaxies have had their star formation quenched, and blue galaxies are still forming stars. However, a nonnegligible fraction of red galaxies are clearly edge-on disk objects that owe their color to an enhanced extinction, and a small fraction of blue galaxies might have already stopped their activity (see, e.g., Bamford et al. 2009; Schawinski et al. 2009).

The bimodality can originate both from a priori differences set beforehand, the so-called nature scenario, and from environmentally driven processes taking place during the evolution of galaxies, the so-called nurture scenario. As discussed in De Lucia et al. (2012), trying to separate the two frameworks and differentiate their role in driving galaxy evolution might be an illposed task because they are strongly and physically connected. According to the $\Lambda$ cold dark matter model, as time goes by, smaller structures merge to form progressively larger ones. This hierarchical growth implies that the fraction of galaxies located in groups progressively increases since $z \sim 1.5$, and at $z \sim 0$ most galaxies are found in groups (Huchra \& Geller 1982; Eke et al. 2004; Berlind et al. 2006; Knobel et al. 2009). It is therefore important to understand the role of the group environment in boosting galaxy transformations from blue to red colors and from late- to early-type morphologies. Color and morphological fractions are very different functions of environment at low $z$ (Bamford et al. 2009). Both are sensitive to stellar mass, but at fixed stellar mass, color is also highly sensitive to environment, while morphology displays much weaker environmental trends (see also Kauffmann et al. 2004; Blanton et al. 2005; Christlein \& Zabludoff 2005; Weinmann et al. 2009; Kovač et al. 2010).

The existence of a variety of "subpopulations" of galaxies whose color does not correspond to what is expected based on their morphology (red late types, blue early types, and so on) suggests that galaxy transformations from blue to red must occur on significantly shorter timescales than transformations from late to early type.

However, what drives the observed trends is still not fully understood. Numerous processes may be responsible for the dependence of galaxy properties on environment (Boselli \& Gavazzi 2006 and references therein). The extreme local 
densities reached within cluster cores enable efficient ram pressure stripping of the galaxy cold gas on timescales of a few megayears (Gunn \& Gott 1972; Abadi et al. 1999). On the other hand, galaxy-group interactions like "strangulation" can remove warm and hot gas from a galaxy halo, efficiently cutting off the star-formation gas supply (Larson et al. 1980; Cole et al. 2000; Balogh et al. 2000a; Kawata \& Mulchaey 2008). Halos can play a role through tidal forces and dynamical friction. Galaxy-galaxy harassment at the typical velocity dispersion of bound groups and clusters may also result in star-formation quenching (Moore et al. 1996). Shock heating in massive halos can prevent accretion of cold gas that would feed star formation. Interactions and mergers can also apply torques that drive gas inward, perhaps feeding and then exhausting star formation or a central black hole.

Radial trends of galaxy properties (e.g., colors, morphologies) as a function of distance from the halo center can be observable effects of these processes responsible for the galaxy transformations. They have been extensively studied in galaxy clusters, where, e.g., a strong radial dependence in the star-formation rate (SFR) is observed (Hashimoto \& Oemler 1999; Balogh et al. 1999; Lewis et al. 2002; Balogh et al. 2004; Tanaka et al. 2004; von der Linden et al. 2010).

Another way to gain insight into the physical processes is to study those galaxies whose morphological type places them on one side of the bimodality but whose star formation identifies them with the other. Blue early-type galaxies with high current SFRs $\left(0.5<\mathrm{SFR}<50 M_{\odot} \mathrm{yr}^{-1}\right)$ or recently stopped starformation activity are one example (e.g., Kannappan et al. 2009; Ferreras et al. 2009; Schawinski et al. 2009). These galaxies tend to live in lower density environments than red sequence early types and make up $\sim 6 \%$ of the low $-z$, general field, early-type galaxy population. They might be early-type galaxies previously on the red sequence that are undergoing an episode of star formation because of the sudden availability of cold gas ("rejuvenated"), making them leave the red sequence before rejoining it. The gas might become available after a merger. If merging occurs between gas-rich galaxies, it may produce a larger amount of star formation (wet mergers) and transform disk galaxies into elliptical galaxies (Lin et al. 2008).

Another example of objects in transition is given by red late types. Their distribution displays a clear trend with both local density and group-centric distance: their fraction increases with increasing local density or decreasing group-centric distance, but at very high densities or in the cores of groups, the red late-type fraction declines sharply (Bamford et al. 2009; Masters et al. 2010; van der Wel et al. 2009). Many of them have some ongoing star formation and are reddened by dust extinction (e.g. Gallazzi et al. 2009; Wolf et al. 2009). They might be the result of quite gentle processes (e.g., galaxy-galaxy interactions, interaction with the intergalactic medium, harassment, strangulation, bar instabilities) that might allow the existence of the spiral structure even shutting down the star formation (e.g., Walker et al. 1996; Skibba \& Sheth 2009; Skibba et al. 2009).

The aim of this study is to characterize in detail the incidence of galaxies of different types in the local universe and depict objects in transition, the analysis of which will help us to shed light on the processes acting on galaxies and the timescale needed for galaxies to transform from one type to the other.

First, we study colors and morphologies of galaxies in different environments. Because many galaxy characteristics are interrelated (Cowie et al. 1996; Gavazzi et al. 1996; Blanton et al. 2003; Kauffmann et al. 2003; Brinchmann et al. 2004; Baldry et al. 2004), we study the correlations for each property independently while fixing other variables. When constraining environmental effects, we perform the analysis in different galaxy stellar mass bins. Galaxy colors and morphologies also correlate, so we split galaxies simultaneously by color and morphological types to distinguish between processes that affect SFRs and structural properties differently. Second, we focus on objects in transition and study in detail their properties, with the aim of understanding the evolutive scenarios of these galaxies.

The analysis has been carried out using a cosmology with $\left(\Omega_{m}, \Omega_{\Lambda}, h\right)=(0.3,0.7,0.7)$, Vega magnitudes (unless otherwise stated), and a Kroupa (2001) initial mass function (IMF).

\section{THE DATA SET AND DATA SAMPLE}

We use the Padova Millennium Galaxy and Group Catalog (PM2GC; Calvi et al. 2011), consisting of a spectroscopically complete sample of galaxies at $0.03 \leqslant z \leqslant 0.11$ brighter than $M_{B}=-18.7$. This sample is sourced from the Millennium Galaxy Catalog (MGC; Liske et al. 2003; Driver et al. 2005), a $B$-band contiguous equatorial survey of $\sim 38 \mathrm{deg}^{2}$ complemented by a $96 \%$ spectroscopically complete survey down to $B=20$, and it is representative of the general field population in the local universe.

By applying a friends-of-friends (FoF) algorithm, Calvi et al. (2011) identified 176 galaxy groups with at least three members with $M_{B}<-18.7$ in the redshift range $0.04 \leqslant z \leqslant 0.1$. A galaxy is considered a group member if its spectroscopic redshift lies within $\pm 3 \sigma$ (velocity dispersion) from the median group redshift and if it is located within a projected distance of $1.5 R_{200}$ from the group geometrical center, where $R_{200}{ }^{6}$ is defined as the radius delimiting a sphere with interior mean density 200 times the critical density of the universe at that redshift and is commonly used as an approximation of the group virial radius. Galaxies that have no neighbors or just one with a projected mutual distance of $0.5 \mathrm{~h}^{-1} \mathrm{Mpc}$ and a redshift within $1500 \mathrm{~km} \mathrm{~s}^{-1}$ are considered "single" or "binary-system" galaxies, respectively.

Applying an FoF to the De Lucia \& Blaizot (2007) semianalytic model (Vulcani et al. 2014), we found that $80 \%$ of our group/binary systems/single galaxies span a halo mass range of $10^{12}-10^{14} M_{\odot} / 10^{11.4}-10^{12.6} M_{\odot} / 10^{11.2}-10^{12.3} M_{\odot}$.

Rest-frame absolute magnitudes are computed using INTERREST (Taylor et al. 2009) from the observed Sloan Digital Sky Survey (SDSS) photometry. The code uses a number of template spectra to carry out the interpolation from the observed photometry in bracketing bands (see Rudnick et al. 2003). Rest-frame colors are derived from the interpolated rest-frame apparent magnitudes.

Stellar masses are estimated following the Bell \& de Jong (2001) relation (Calvi et al. 2011), which correlates the stellar mass-to-light ratio with the optical colors of the integrated stellar population, using the $B$-band photometry taken from the MGC and the rest-frame $B-V$ color computed from the Sloan $g-r$, color corrected for Galactic extinction:

$$
\log _{10}\left(M / L_{B}\right)=-0.51+1.45(B-V),
$$

valid for a Bruzual \& Charlot model with solar metallicity and a Salpeter $(1955)$ IMF $\left(0.1125 M_{\odot}\right)$. Then they are converted to a

\footnotetext{
6 The $R_{200}$ values are computed from the velocity dispersions as in Finn et al. (2005).
} 
Kroupa (2001) IMF, adding -0.19 dex to the logarithmic value of the masses (Cimatti et al. 2008). The typical uncertainty in mass estimates is 0.20.3 dex (for details and comparisons with external estimates, refer to Calvi et al. 2011; Poggianti et al. 2013). The sample is complete for $\log M_{\star} / M_{\odot}>10.25$, corresponding to the mass of the faintest and reddest galaxy $\left(M_{B}=-18.7, B-V=0.9\right)$ at our redshift upper limit $(z=0.1)$, as described in Calvi et al. (2012).

SFRs and star-formation histories (SFHs) are derived by fitting the spectra with the spectrophotometric model fully described in Fritz et al. (2007, 2011). The MGC spectroscopic database of PM2GC galaxies consists of SDSS, 2dFGRS, and MGCz spectra (Driver et al. 2005), the latter taken with the same instrument and setup as the 2dFGRS. Always choosing the highest quality spectrum available, SDSS spectra are preferred when possible ( $86 \%$ of the sample), alternatively 2dFGRS spectra when available (12\%), and MGCz spectra in the remaining cases. In the Fritz et al. (2007) model, all of the main spectrophotometric features (i.e., the continuum flux and shape, the equivalent widths of emission and absorption lines) are reproduced by summing the theoretical spectra of simple stellar populations (SSP) of 12 different ages (from $3 \times 10^{6}$ to $\sim 14 \times 10^{9} \mathrm{yr}$ ). The spectral analysis allows us to derive an estimate of the SFRs at different cosmic times and of the average age of the stars in a galaxy.

Morphologies are determined using MORPHOT (Fasano et al. 2012), an automatic tool designed to reproduce as closely as possible the visual classifications. MORPHOT adds to the classical CAS (concentration/asymmetry/clumpiness) parameters a set of additional indicators derived from digital imaging of galaxies and has been proved to give an uncertainty only slightly larger than the eyeball estimates. It was applied to the $B$-band MGC images to identify ellipticals, lenticulars (S0s), and later-type galaxies (Calvi et al. 2012).

Structural parameters are taken from the public catalog of the MGC database (Allen et al. 2006) and were derived from a two-dimensional surface-brightness profile fitting of MGC-BRIGHT galaxies using GIM2D (Simard et al. 2002). We use the catalog obtained in adopting a bulge-disk decomposition model using a Sérsic bulge plus exponential disk. As described in Allen et al. (2006), the components were required to have a common spatial center, but their luminosities were independent, allowing the calculation of a bulge-to-total (B/T) luminosity ratio for each galaxy. We refer readers to the original paper for additional details.

In this paper, we consider two galaxy classes and treat separately the most massive galaxy (MMG) of each structure and all of the other galaxies, called satellites, regardless of environment. With this definition, all single galaxies are treated as MMGs, and binary systems are split into one MMG and one satellite. In addition, we remove active galactic nuclei (AGNs) because their presence might alter the results. To do that, we match our sample with the latest AGN catalog from SDSS, ${ }^{7}$ finding that overall 48 galaxies that enter our entire masscomplete sample are indeed hosting an AGN. Applying the fraction of AGNs obtained $(<4 \%)$ to the PM2GC subsample without spectra from SDSS, we expect $\sim 9$ AGNs not detected above our mass completeness limit. We assume that this number is negligible.

The final mass-complete sample of galaxies in groups consists of 417 satellites and 165 MMGs. The final binary-system and

\footnotetext{
7 https://www.sdss3.org/dr10/spectro/spectro_access.php
}

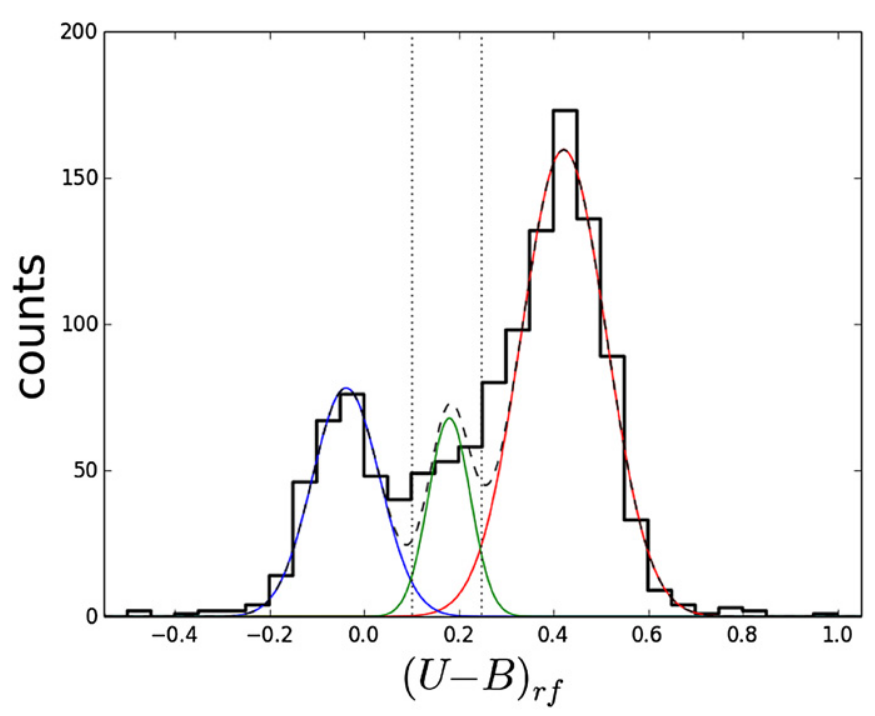

Figure 1. Rest-frame $(U-B)_{r f}$ color distribution of all galaxies in our masscomplete sample. Also plotted are Gaussian fits to the red, blue, green (solid lines), and total (black dashed line) population. The vertical lines indicate where Gaussian fits intersect.

single-galaxy samples consist of 228 (170 of which are MMGs) and 418 galaxies, respectively.

\section{GALAXY SUBPOPULATIONS}

Broadly speaking, based on color, galaxies can be subdivided into two populations: red and blue. To start, we plot the $(U-B)_{r f}$ distribution of all galaxies above the mass completeness limit. Figure 1 highlights the presence of the well-known bimodality, but also of an emerging third population located between the main peaks. We will refer to these as "green" galaxies. We fit the entire distribution with three Gaussian functions, to define the boundaries of the green population so it has minimal overlap with the red and blue sequences. The position of the peaks depends on mass and redshift, so in principle, we should fit the color distribution at different stellar masses and in different redshift bins and then interpolate to find a relation that depends on stellar mass, color, and redshift. Because no significant evolution is expected between $z \sim 0.1$ and $z \sim 0.04$, we neglect the redshift dependence. Given that our mass range is quite small, we cannot compute the dependence of the cut on stellar mass, and we adopt the slope of the color-mass relation presented in Peng et al. (2010) (0.075). To compute the zero points of the relations that separate different colors, we focus on the mass bin $M_{\star}=10^{10.5-11} M_{\odot}$ and find the values where the blue and green and the green and red Gaussians intersect each other. Galaxies are assigned to the red sequence if their rest-frame color obeys

$$
(U-B)_{\text {Vega }} \geqslant 0.075 \times \log M_{*}-0.561,
$$

to the blue cloud if their rest-frame color obeys

$$
(U-B)_{\text {Vega }} \leqslant 0.075 \times \log M_{*}-0.709,
$$

and to the green valley if they are between the two cuts. This is visually shown in the upper panel of Figure 2, where we also plot galaxies that do not enter our mass-complete sample, just to show the general trends.

Table 1 presents the percentage of galaxies of different types and colors in different environments for the mass-complete 

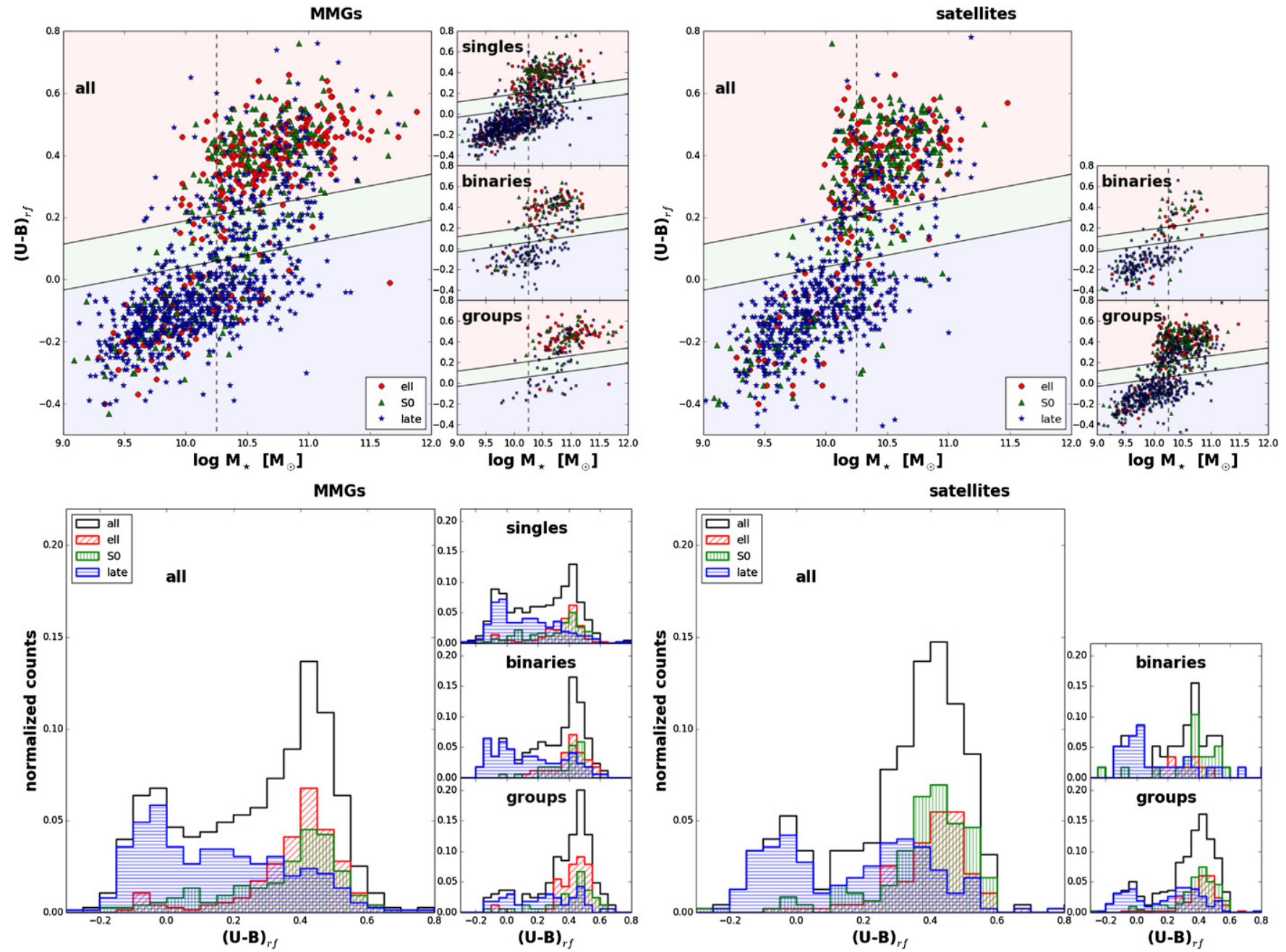

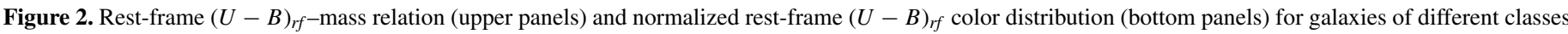

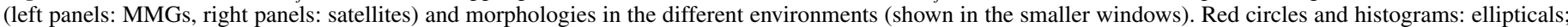

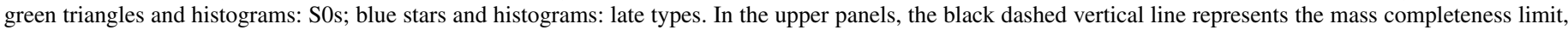
and the black solid lines show the separation between red, green, and blue galaxies (see the text for details).

samples. The majority of galaxies are red, and more so for satellites $(\sim 70 \%)$ than MMGs $(\sim 60 \%)$. Green galaxies are $14 \%$ of all MMGs and $9 \%$ of all satellites, and blue galaxies are $26 \%$ of MMGs and $19 \%$ of satellites.

Color fractions strongly depend on environment (singles, binaries, groups) and not on galaxy class, with that of MMGs and satellites always being compatible within the errors. Interestingly, green galaxies are always about half as numerous as blue galaxies, showing that their relative number is independent both of environment and of the distinction between satellites and MMGs (see Section 4.1 for more details).

Considering morphologies, late types dominate both galaxy classes, being $\sim 40 \%$ and $\sim 50 \%$ of all satellites and MMGs, respectively. For both classes, this fraction decreases in going from less to more massive environments. Ellipticals are instead found in the same proportion among MMGs and satellites. In addition, ellipticals and S0s are found in similar proportions among MMGs, while among satellites S0s are more frequent. These latter trends remain true when individual environments are inspected, except that ellipticals are more numerous than S0s in group MMGs. Thus, at odds with the color fractions, morphological fractions vary both between groups, binaries, and singles and from MMGs to satellites in a given environment.
The bottom panels of Figure 2 show that even though a color bimodality exists for both MMGs and satellites, their color distributions are remarkably different: they both show a prominent red peak at $(U-B)_{r f} \sim 0.45$ and a secondary blue peak at $(U-B)_{r f} \sim-0.05$. Nonetheless, blue and green galaxies are more conspicuous among MMGs than among satellites, suggesting that only the blue fractions depend on environment and class. Going from the least toward the most massive environments, the two peaks of the distributions shift toward redder colors, the peak at blue colors becomes less prominent, and the number of galaxies with red color increases, indicating again that in groups, galaxies are most likely red. This is valid for both MMGs and satellites.

Splitting galaxies by morphology, among MMGs, late types show a unimodal distribution with a long tail toward green and red colors, but among satellites they present a bimodal distribution, with a second peak almost as important as the first one, centered at quite red colors, and only a few green galaxies. Late types are the galaxies that show the most noticeable variation with the galaxy class and environment. Ellipticals and S0s are mostly red, but green and blue ellipticals and S0s are a nonnegligible fraction. In MMGs, ellipticals and S0s show similar color distributions peaked around $(U-B)_{r f} \sim 0.4$. Both distributions show a tail of galaxies with blue colors. In satellites, 
Table 1

Percentage of Galaxies of Different Types Above the Stellar Mass Completeness Limit in Different Environments

\begin{tabular}{|c|c|c|c|c|c|c|c|c|c|c|c|c|c|}
\hline & & \multicolumn{6}{|c|}{ Most Massive Galaxies } & \multicolumn{6}{|c|}{ Satellites } \\
\hline & & \multicolumn{3}{|c|}{ Color } & \multicolumn{3}{|c|}{ Morphology } & \multicolumn{3}{|c|}{ Color } & \multicolumn{3}{|c|}{ Morphology } \\
\hline & & Red & Green & Blue & Ell & So & Late & Red & Green & Blue & Ell & So & Late \\
\hline \multicolumn{14}{|c|}{ All Galaxies } \\
\hline \multirow{4}{*}{ Color } & All & $60 \pm 3$ & $14 \pm 2$ & $26 \pm 2$ & $28 \pm 2$ & $25 \pm 2$ & $47 \pm 3$ & $72 \pm 3$ & $9 \pm 2$ & $19 \pm 3$ & $25 \pm 3$ & $35 \pm 3$ & $\overline{40 \pm 3}$ \\
\hline & Red & & & & $25 \pm 2$ & $19 \pm 2$ & $16 \pm 2$ & & & & $23 \pm 3$ & $30 \pm 3$ & $19 \pm 3$ \\
\hline & Green & & & & $1.2 \pm 0.6$ & $3 \pm 1$ & $9 \pm 2$ & & & & $1 \pm 1$ & $3 \pm 1$ & $5 \pm 1$ \\
\hline & Blue & & & & $2 \pm 1$ & $3 \pm 1$ & $21 \pm 2$ & & & & $1 \pm 1$ & $2 \pm 1$ & $16 \pm 2$ \\
\hline \multicolumn{14}{|c|}{ Single Galaxies } \\
\hline \multirow{4}{*}{ Color } & All & $53 \pm 3$ & $16 \pm 3$ & $31 \pm 2$ & $24 \pm 3$ & $26 \pm 3$ & $50 \pm 3$ & $\ldots$ & $\ldots$ & $\ldots$ & $\ldots$ & $\ldots$ & $\ldots$ \\
\hline & Red & & & & $20 \pm 3$ & $17 \pm 3$ & $16 \pm 2$ & & & & $\ldots$ & $\cdots$ & $\ldots$ \\
\hline & Green & & & & $1 \pm 1$ & $4 \pm 1$ & $10 \pm 2$ & & & & $\ldots$ & $\ldots$ & $\ldots$ \\
\hline & Blue & & & & $3 \pm 1$ & $4 \pm 1$ & $24 \pm 3$ & & & & $\ldots$ & $\ldots$ & $\ldots$ \\
\hline \multicolumn{14}{|c|}{ Binary Sytems } \\
\hline \multirow{4}{*}{ Color } & All & $59 \pm 5$ & $14 \pm 4$ & $27 \pm 5$ & $26 \pm 5$ & $21 \pm 5$ & $53 \pm 6$ & $57 \pm 9$ & $12 \pm 6$ & $31 \pm 9$ & $17 \pm 8$ & $36 \pm 9$ & $47 \pm 9$ \\
\hline & Red & & & & $23 \pm 5$ & $19 \pm 5$ & $17 \pm 4$ & & & & $12 \pm 7$ & $29 \pm 9$ & $15 \pm 7$ \\
\hline & Green & & & & $2 \pm 2$ & $0.5 \pm 0.1$ & $11 \pm 4$ & & & & $5 \pm 5$ & $2 \pm 2$ & $5 \pm 5$ \\
\hline & Blue & & & & $0.6 \pm 0.1$ & $1 \pm 1$ & $25 \pm 5$ & & & & $0_{-0}^{+1}$ & $5 \pm 5$ & $26 \pm 9$ \\
\hline \multicolumn{14}{|c|}{ Groups } \\
\hline \multirow{4}{*}{ Color } & All & $79 \pm 5$ & $7 \pm 3$ & $14 \pm 4$ & $42 \pm 6$ & $26 \pm 5$ & $32 \pm 5$ & $74 \pm 3$ & $9 \pm 2$ & $17 \pm 3$ & $26 \pm 3$ & $34 \pm 3$ & $40 \pm 3$ \\
\hline & Red & & & & $40 \pm 6$ & $22 \pm 5$ & $16 \pm 4$ & & & & $24 \pm 3$ & $30 \pm 3$ & $20 \pm 3$ \\
\hline & Green & & & & $0_{-0}^{+1}$ & $2 \pm 1$ & $5 \pm 2$ & & & & $1 \pm 1$ & $3 \pm 1$ & $5 \pm 2$ \\
\hline & Blue & & & & $2 \pm 2$ & $2 \pm 2$ & $10 \pm 4$ & & & & $1 \pm 1$ & $2 \pm 1$ & $15 \pm 2$ \\
\hline
\end{tabular}

instead, S0s are the most important population forming the red peak.

This analysis highlights the variation of the incidence of each subpopulation with environment and the well-known fact that there is no one-to-one correspondence between color and morphology, i.e., not all late types have blue colors, and not all ellipticals and SOs are red.

In the following we refer to red late types, blue early types, and green galaxies of all types as candidate "transition objects" because they are likely to have experienced, or are in the process of experiencing, a transformation in color and/or morphology from star forming to passive, or vice versa.

Until now we have considered only galaxy colors and morphologies, but these do not univocally distinguish between star-forming and passive galaxies. The red late-type sample might be contaminated by the presence of star-forming galaxies highly extincted by dust ${ }^{8}$ and, conversely, not all blue or green early types are necessarily forming stars. To isolate truly star-forming and truly passive galaxies, we inspect the level of specific star-forming rate $(\mathrm{SSFR})\left(=\mathrm{SFR} / M_{\star}\right){ }^{9}$ From now on, we only consider red late-type objects with SSFR $<10^{-12} \mathrm{yr}^{-1}$ (RP late types) and blue early-type objects with SSFR > $10^{-12} \mathrm{yr}^{-1}$ (BSF early types).

\footnotetext{
8 We remind the reader that the Fritz et al. (2007) model includes a treatment of the dust, so in principle our derived SSFRs already take into account the possible presence of dust and are corrected for that. In practice, models are not able to detect highly extincted star formation in the optical without the aid of infrared data. The levels of SFRs in our sample, however, are not expected to be extreme in the vast majority of cases, so our dust treatment and SFRs can be considered overall reliable.

9 The typical relative error on the SSFR is $35 \%-40 \%$.
}

\section{TRENDS WITH MASS AND ENVIRONMENT}

\subsection{Mass Trends in Different Environments}

Calvi et al. $(2012,2013)$ have already shown that in the PM2GC galaxy, morphology is linked with both stellar mass and environment. The mass distribution of each morphological type depends on the environment, and in each environment the mass function is different for ellipticals, S0s, and late types. They found that there is little dependence of the morphological fractions on galaxy mass in the range $10.25<M<11.1$, but, at higher masses, this dependence is strong.

Here we take a complementary approach, also carefully inspecting the changes in color. First, we apply only the cut in color for MMGs and satellites separately (Figure 3). Fractions strongly depend both on stellar mass and class. As shown in the left panels, the contribution of blue galaxies to the total population steadily decreases with mass. However, at any mass, blue galaxies are more numerous among MMGs than among satellites, being $40 \%$ of the entire MMG population at $\log M_{\star} / M_{\odot}=10.4$, and $<25 \%$ of satellites at the same mass. Also, the fraction of green galaxies decreases with increasing mass, for both MMGs and satellites, though more gently than in blue galaxies. In contrast, the incidence of red galaxies increases with mass, and they dominate both galaxy classes at all masses.

Environmental variations of color fractions can also be observed comparing group and single MMGs: red/blue and green galaxies are less/more frequent among singles than in groups at a given mass. No significant differences are detected between group satellites and all satellites, as expected given the fact that $90 \%$ of our satellites are in groups. On the other hand, we note that considering only groups, color fractions as a function of mass are very similar for MMGs and satellites, 


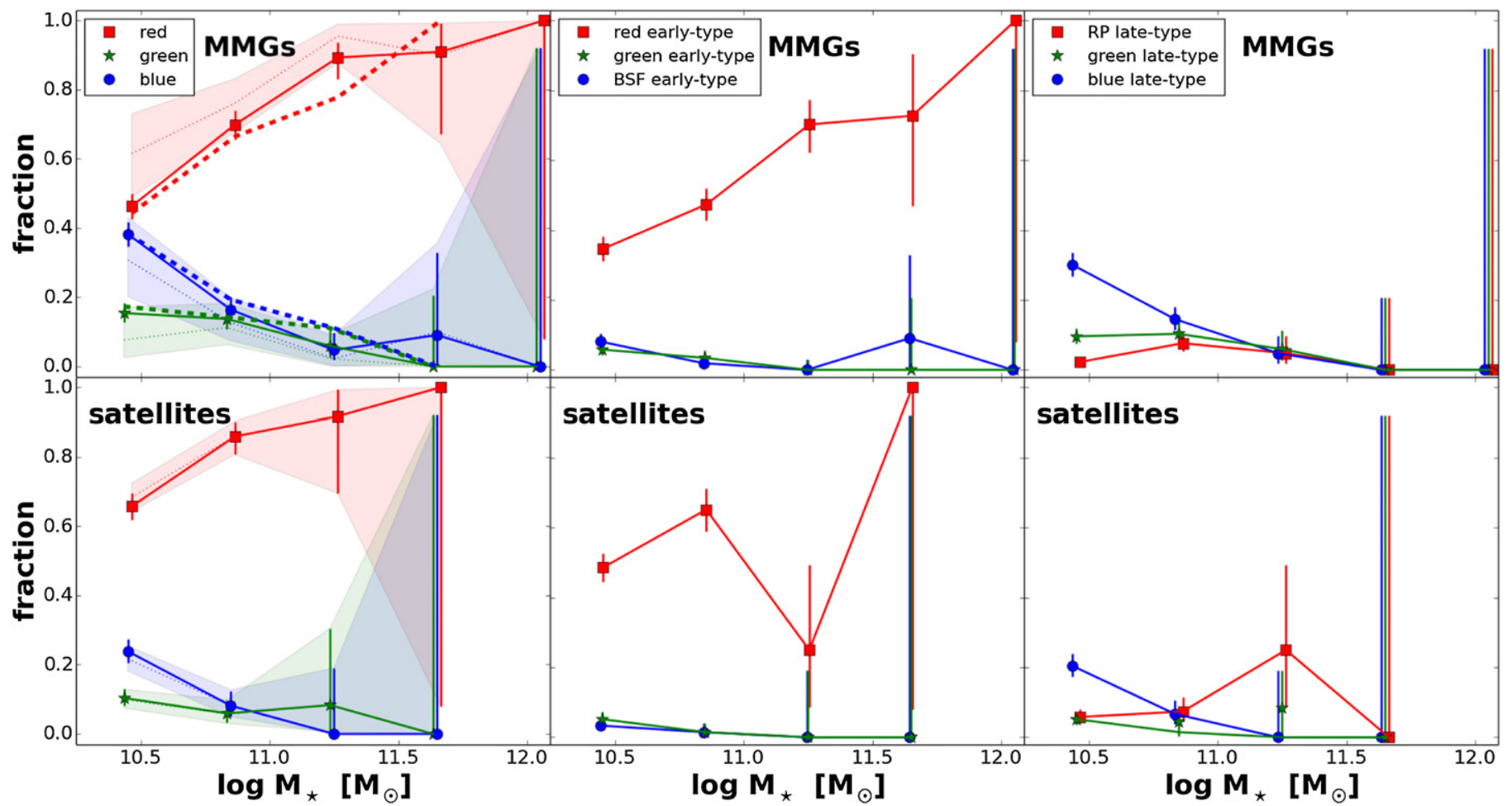

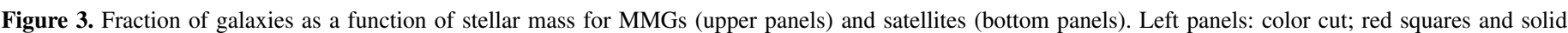

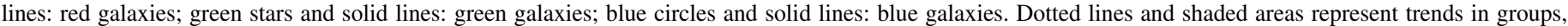

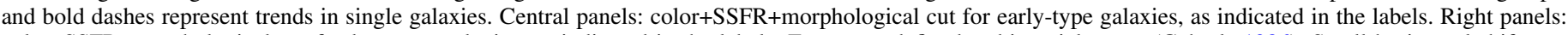

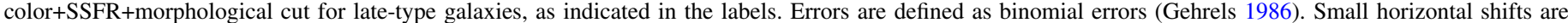
applied to make the plot clearer.

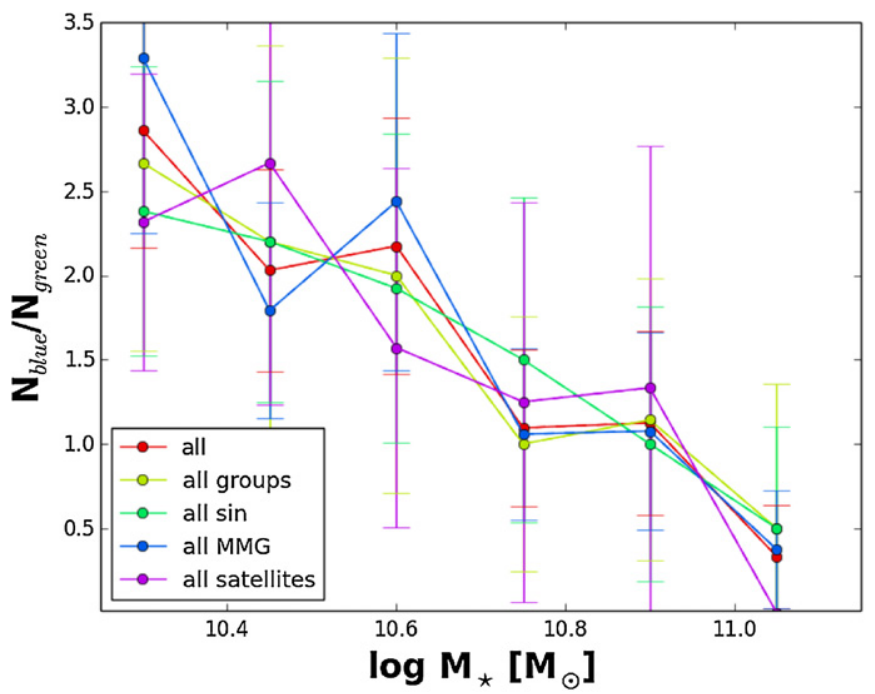

Figure 4. Ratio of the number of blue galaxies to the number of green galaxies as a function of stellar mass, for galaxies in different environments and of different class.

indicating that differences between all MMGs and all satellites of a given mass are driven by the single MMGs.

More interesting than the simple green fraction is the number of green galaxies relative to the number of blue galaxies, which probably are evolutionarily linked. Figure 4 presents the ratio of blue to green galaxies as a function of mass for all galaxies, for single and group galaxies and for MMGs and satellites. Trends are very similar in all cases, with the blue-to-green ratio always a factor of 1:2 at high masses, 1:1 at intermediate masses, and
2.53:1 at lower masses. We will discuss the meaning of this ratio later, when we discuss in detail the evolutionary links between green and blue galaxies.

We now apply a joint subdivision in color, morphology, and SSFR (central and right panels in Figure 3). Here and in the following figures, we consider together elliptical and S0 galaxies (early types), in order to increase the statistics and improve the readability of the panels. ${ }^{10}$ While the fraction of red early types increases with mass, ${ }^{11}$ those of BSF and green early types tend to decrease and are very close to zero at high masses. Also, the trends for blue, green, and RP late types are different, steadily declining in the former and consistent with being flat with mass in the others. This is true both for MMGs and for satellites. Moreover, RP late types, BSF, and green early-type trends with mass do not change with environment (groups versus singles, plots not shown).

In Section 7 we will analyze in detail transition galaxies, but we first focus on trends in groups, where galaxy properties might also be driven by the location of the galaxies within the group.

\subsection{Radial Trends in Groups}

In groups there might be an additional parameter that can drive galaxy transformations: the position of galaxies within their structure. Indeed, galaxies are expected to fall toward halo centers because of dynamical friction, so their group-centric

\footnotetext{
10 We note that for $\log M_{\star} / M_{\odot}>11$ ellipticals are twice as numerous as S0s (see also Calvi et al. 2012).

11 The observed dip in satellite red early types is probably due to galaxies with a consistent bulge but that have been classified as late types and to small-number statistics. Indeed, in Appendix A, where trends are inspected considering the Sérsic index instead of morphology, the fraction of red bulges steadily increases with mass.
} 


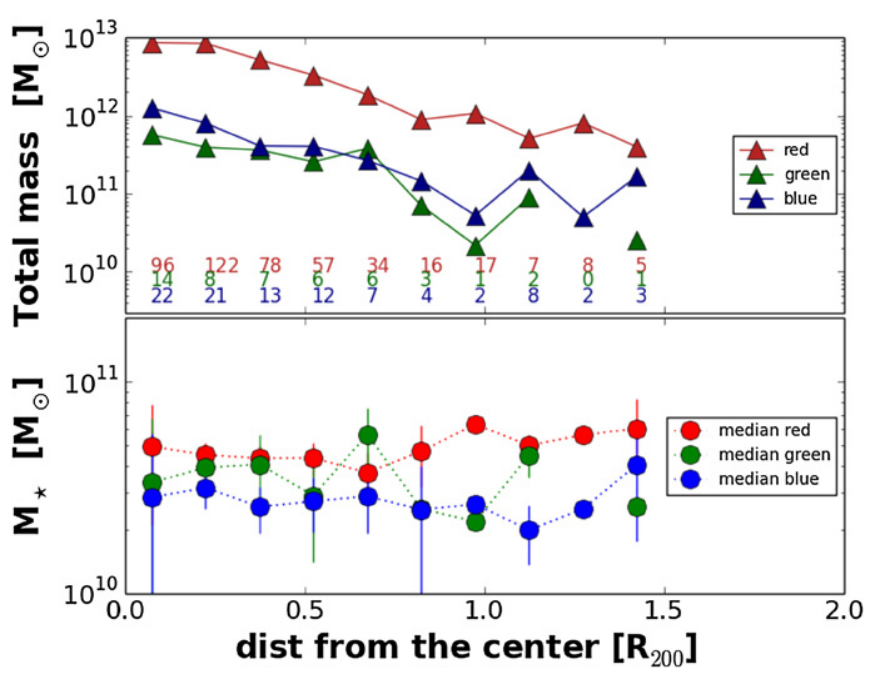

Figure 5. Total (upper panel) and median (bottom panel) stellar mass as a function of group-centric distance for red, green, and blue MMG+satellite galaxies in groups above the mass completeness limit. Numbers are the number of objects in each bin.

distance is related to the time that galaxies have spent inside the group (Weinmann et al. 2011; Smith et al. 2012; De Lucia et al. 2012).

We first look for signs of mass segregation with distance. Considering MMGs and satellites together, we sum the stellar mass of all galaxies (total mass). Figure 5 shows that for red, green, and blue galaxies, the total mass in galaxies depends on group-centric distance: there is more mass close to the group center than in the outskirts because of the larger number of galaxies at small group-centric distances. However, the median stellar mass is independent of distance for galaxies of any color separately, as shown in the bottom panel. Fluctuations in trends of green galaxies are probably due to small-number statistics: at $r>0.7 R_{200}$ there are only seven galaxies. While green and blue galaxies present similar average masses, red galaxies are systematically more massive, by a factor of two.

Therefore, stellar mass and position within groups are not strictly related and might play different roles in driving galaxy transformations. To separate the two contributions, we investigate how fractions depend on group-centric distance in bins of stellar mass.

We start by analyzing galaxy color fractions in groups and comparing them to binary and single systems. Figure 6 shows qualitatively similar trends for MMGs and satellites, although satellites have better statistics. At low masses (upper left panel), red galaxies dominate at small group-centric distances, while in the outskirts they are as common as blue galaxies, whose fraction increases with distance. The fraction of green galaxies is consistent within the errors with being constant with distance. At higher masses (lower left panel) all trends are flat, with red galaxies always representing $\sim 75 \%-80 \%$ of the total population. In both mass bins, in binaries, galaxies of any color are, within the errors, about as common as galaxies of the same color in the group outskirts. The effect of environment is visible only among single galaxies for an excess of blue objects at high masses.

Considering also morphologies and star-forming properties, uncertainties increase, but some trends are still robustly detected. At low masses (upper central and right panels in Figure 6), red early types dominate the populations, but their percentage slightly decreases with distance. In MMGs their de- crease is mirrored by BSF early types, in satellites by blue late types. The fraction of RP late types and green early types and late types is flat with distance. At higher masses, all trends are flat, with a possible excess of RP late types in the outskirts for MMGs. We do not detect any significant environmental variation.

\subsection{Possible Systematics}

There are several possible biases or other effects in the data to consider in order to ensure the robustness of these results.

First of all, we note that any contamination of the group sample by field galaxies and vice versa, for which we are not applying any correction, will only render the observed trends less prominent. The real, corrected trends therefore would be even more pronounced.

As described in Calvi et al. (2011), some of the groups are not fully contained in the narrow strip of the MGC survey and hence suffer from edge problems. Fifty of these groups enter our sample, for a total of 313 galaxies. Removing them from our analysis, all of the trends are recovered within the errors (plots not shown). Because our results are not affected by these groups, we keep them in our sample to have better statistics.

Our groups show a variety of galaxy richness: the smallest systems host only three members, the biggest one about 60 (Calvi et al. 2011). As a consequence, the results might be influenced by the different numbers of galaxies in groups, so we again performed our analysis, splitting galaxies into two bins of richness: those in groups with $N_{\text {gal }}<7$ members and those hosted in larger systems. ${ }^{12}$ This cut allows us to have almost equally numerous bins. No signs of a grouprichness dependence are evident, except for the fact that richer groups tend to have more massive MMGs. All MMGs in the richest groups are more massive than $\log M_{\star} / M_{\odot}=10.7$ (plots not shown).

We also note that the uncertainty associated with the determination of the group centers might be quite large, especially for groups composed of only three objects. Once again, the real, corrected trends would be more noticeable.

In our analysis, we have distinguished between MMGs and satellites. However, MMGs do not always coincide with the galaxy closest to the group geometrical center. MMGs are mainly located in the central regions of the halos $(74 \%$ are within $r=0.5 R_{200}$ ), but not always at their very center ( $34 \%$ also correspond to the galaxy closest to the geometrical center). ${ }^{13}$ This can be understood as different stages in the hierarchical clustering process (Brough et al. 2008; Pimbblet 2008). We note that this fraction is in agreement with that estimated by Skibba et al. (2011), who computed the fraction of brightest noncentral galaxies as a function of the halo mass, finding that it is $25 \%$ in low-mass halos $\left(10^{12} h^{-1} M_{\odot} \leqslant M \leqslant 2 \times 10^{13} h^{-1} M_{\odot}\right)$, and it increases with halo mass. Our choice of contrasting MMGs and all other galaxies (common to many studies, e.g., Weinmann et al. 2006, 2009; Skibba et al. 2007; van den Bosch et al. 2008; Pasquali et al. 2009, 2010) most likely produces an underestimate of the true differences between centrals and satellites, so the trends might be even more pronounced.

\footnotetext{
12 The cut is applied considering all members and not only those above the mass completeness limit.

13 In a few cases they are on the edge of the groups, though at least three MMGs at $r>1 R_{200}$ are found in groups that might suffer from edge problems.
} 
MMGs

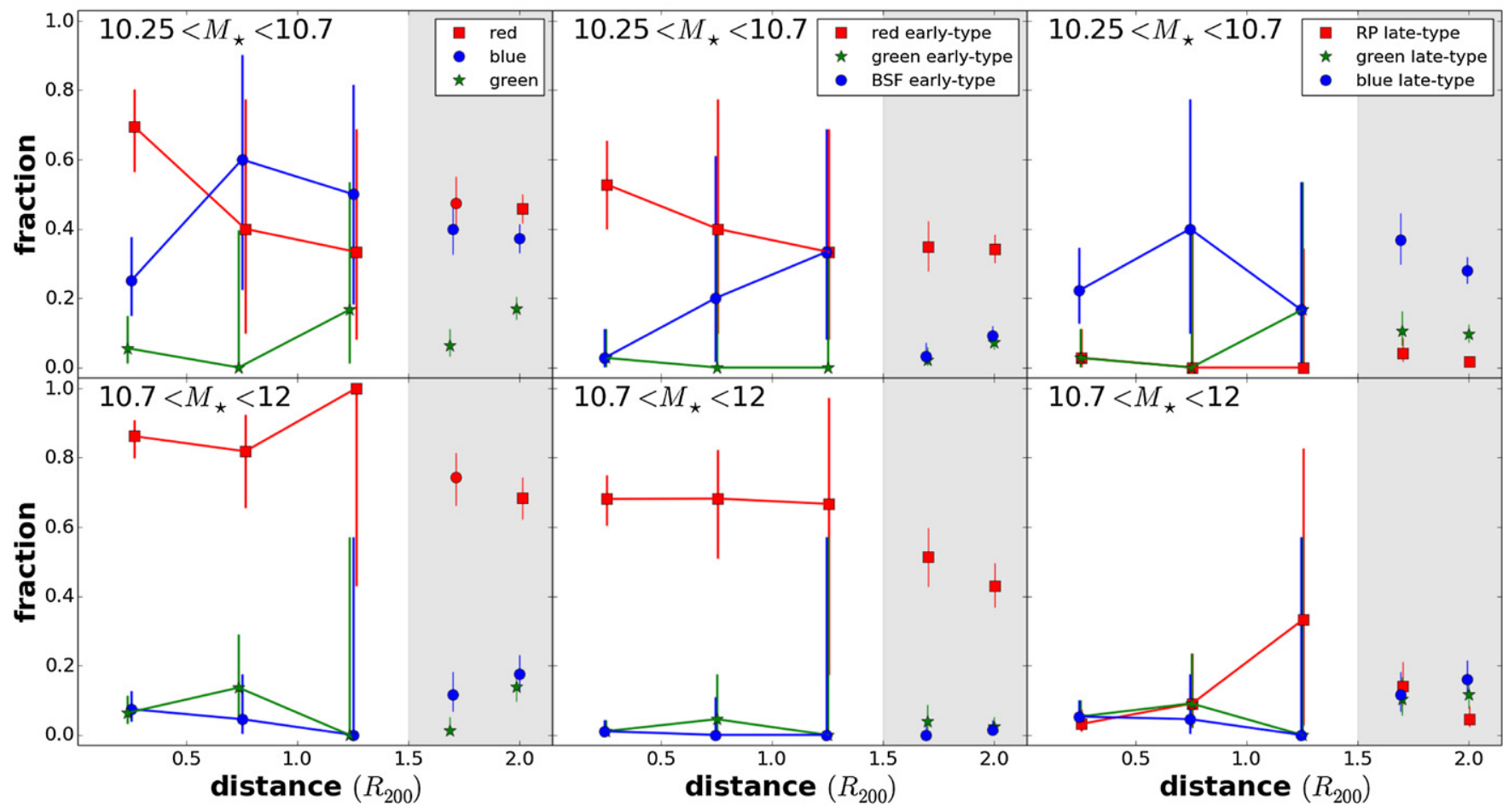

satellites

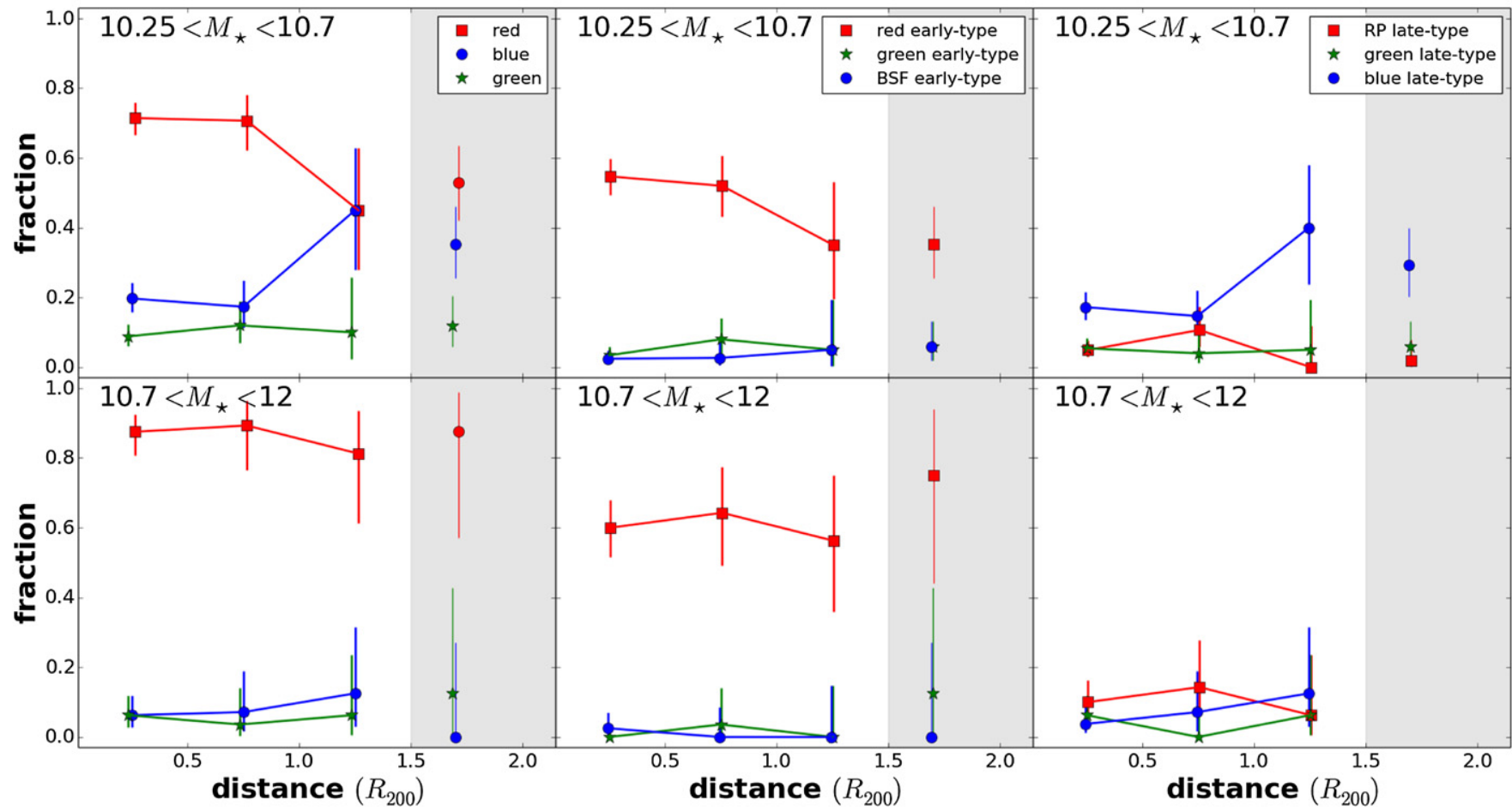

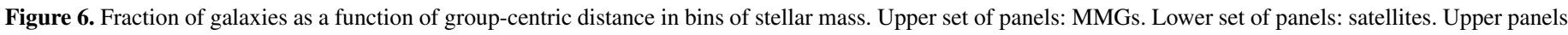

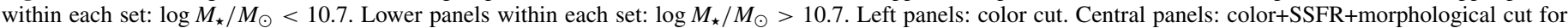

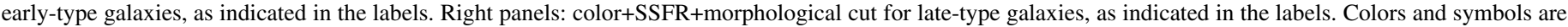

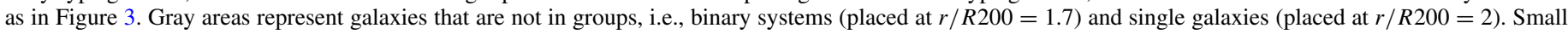
horizontal shifts are applied to make the plot clearer.

\section{OBJECTS IN TRANSITION}

In the previous sections, we have investigated the color and morphological fractions as a function of stellar mass and environment. This analysis has emphasized the presence of objects likely in transition from one type to the other. Above our mass completeness limit, $4 \% \pm 1 \%$ of galaxies are BSF early types ( $2 \% \pm 1 \%$ of MMGs, $8 \% \pm 2 \%$ of satellites), $4 \% \pm 1 \%$ 
Table 2

Characteristic Numbers of Objects in Transition, Compared to Normal Galaxies of the Same Morphology

\begin{tabular}{|c|c|c|c|c|c|c|}
\hline \multirow[t]{2}{*}{ Quantity } & \multicolumn{2}{|c|}{ Red } & \multicolumn{2}{|c|}{ Green } & \multicolumn{2}{|c|}{ Blue } \\
\hline & Early-type & Passive Late-type & Early-type & Late-type & SF Early-type & Late-type \\
\hline Number & 576 & 62 & 51 & 94 & 50 & 236 \\
\hline$\left\langle B_{\text {Vega }}\right\rangle$ & $-19.93 \pm 0.04$ & $-20.0 \pm 0.1$ & $-19.84 \pm 0.1$ & $-20.03 \pm 0.07$ & $-19.9 \pm 0.1$ & $-20.31 \pm 0.06$ \\
\hline$\left\langle\log M_{\star} / M_{\odot}\right\rangle$ & $10.63 \pm 0.04$ & $10.76 \pm 0.05$ & $10.48 \pm 0.05$ & $10.56 \pm 0.05$ & $10.46 \pm 0.05$ & $10.43 \pm 0.03$ \\
\hline$\langle\mathrm{SFR}\rangle\left(M_{\odot} \mathrm{yr}^{-1}\right)$ & $0 \pm 0$ & $0 \pm 0$ & $1.4 \pm 0.5$ & $2.2 \pm 0.5$ & $2.9 \pm 0.5$ & $3.6 \pm 0.5$ \\
\hline$\langle\mathrm{SSFR}\rangle^{\mathrm{a}}\left(\mathrm{yr}^{-1}\right)$ & $(9 \pm 3) 10^{-12}$ & $(6 \pm 3) 10^{-13}$ & $(4 \pm 2) 10^{-11}$ & $(4.2 \pm 0.8) 10^{-11}$ & $(6 \pm 1) 10^{-11}$ & $(9.4 \pm 0.9) 10^{-11}$ \\
\hline$\langle n\rangle$ & $3.41 \pm 0.08$ & $2.9 \pm 0.3$ & $2.8 \pm 0.3$ & $1.7 \pm 0.2$ & $2.7 \pm 0.3$ & $0.8 \pm 0.1$ \\
\hline$\langle\mathrm{B} / \mathrm{T}\rangle$ & $0.58 \pm 0.01$ & $0.26 \pm 0.04$ & $0.54 \pm 0.06$ & $0.06 \pm 0.03$ & $0.55 \pm 0.06$ & $0 \pm 0$ \\
\hline$\left\langle R_{e}(\mathrm{bulge})\right\rangle(\mathrm{kpc})$ & $1.10 \pm 0.05$ & $1.07 \pm 0.2$ & $1.1 \pm 0.2$ & $0.7 \pm 0.2$ & $1.2 \pm 0.2$ & $0 \pm 0$ \\
\hline$\left\langle R_{e}(\right.$ disk $\left.)\right\rangle(\mathrm{kpc})$ & $2.28 \pm 0.09$ & $3.2 \pm 0.3$ & $2.1 \pm 0.2$ & $3.3 \pm 0.3$ & $2.0 \pm 0.2$ & $3.4 \pm 0.5$ \\
\hline$\langle(u-r) \text { bulge }\rangle^{\mathrm{b}}$ & $2.65 \pm 0.02$ & $2.67 \pm 0.05$ & $2.26 \pm 0.09$ & $2.26 \pm 0.05$ & $2.21 \pm 0.09$ & $1.84 \pm 0.22$ \\
\hline$\langle(u-r) \text { disk }\rangle^{\mathrm{b}}$ & $2.52 \pm 0.04$ & $2.5 \pm 0.1$ & $2.3 \pm 0.1$ & $2.11 \pm 0.05$ & $2.2 \pm 0.1$ & $1.79 \pm 0.02$ \\
\hline
\end{tabular}

Notes.

a Values computed only with galaxies with SSFR $\neq 0$.

${ }^{\mathrm{b}}$ Colors are in the AB system.

are green early types $(4 \% \pm 1 \%$ of MMGs and $4 \% \pm 1 \%$ of satellites), $8 \% \pm 1 \%$ are green late types $(9 \% \pm 1 \%$ of MMGs and $5 \% \pm 1 \%$ of satellites), and $5 \% \pm 1 \%$ are RP late types (4\% $\pm 1 \%$ of MMGs and $6 \% \pm 1 \%$ of satellites).

We now analyze the star-formation levels, ages, and structural parameters of these galaxies. From now on, we show the results for MMGs and satellites together, to increase the statistics, having checked that no substantial differences exist between the two populations when the effect of the different mass distribution is considered.

Figure 7 presents the normalized distributions of the most relevant quantities for the objects in transition, compared to their normal counterparts: $M_{B_{\mathrm{Vega}}}$ magnitudes, stellar masses, SFRs, SSFRs, Sérsic indexes, B/T ratios, $(u-r)$ rest-frame color of bulges and disks, and radii of bulges and disks. Figure 8 presents the same quantities as a function of stellar mass, in order to show that the different mass distributions of the samples are not fully responsible for the differences observed in the stellar population and structural parameters. Table 2 gives the mean values of the aforementioned quantities for all of the different subpopulations. The quoted uncertainties in the median are estimated as $1.253 \sigma / \sqrt{N}$, where $\sigma$ is the standard deviation about the median and $N$ is the number of galaxies in the sample under consideration (Rider 1960).

In the following, we directly compare the properties of the objects in transition to those of the subpopulations that might share a similar history with them.

\subsection{Blue Star-forming Early Types}

We now compare BSF early types to red early types and blue late types (Figure 7 and left panels of Figure 8). Because ellipticals and SOs are expected to be characterized by different properties, in Appendix B we report the differences between these two populations.

The mass distribution of BSF early types is very similar to blue late types, while red early types are systematically more massive. At any given mass, BSF early types are on average brighter than their red counterparts, but fainter than blue late types.

The SFR-mass (and SSFR-mass) relations are similar for BSF early types and blue late types, but that of BSF early types is truncated at $\mathrm{SFR} \sim 10 M_{\odot}$, while that of blue late types is not. In contrast, the SFR-mass relation for the subset of red early types that are star-forming is well below the standard relation at all masses.

BSF early types resemble their red counterparts in the $n$-mass plane. They span the whole range of $\mathrm{B} / \mathrm{T}$ ratios, from pure disks to pure bulges. Both their $n$ and $B / T$ distributions are very different from those of blue late types.

The bulge and disk colors of BSF early types are between those of their red counterparts and blue late types. The bulges lie on the upper edge of the corresponding blue late-type color-mass relation, and most of the disks lie just above it. In addition, although most of the bulges follow the same mass-size relation in BSF and in red early types, we detect a subpopulation that is more than an order of magnitude smaller. This bimodality in bulge size is also seen in the BSF late-type population, where many galaxies have no bulge. Disks in BSF galaxies have sizes similar to those in red galaxies and trace the lower edge of the blue late-type relation.

We also analyzed the concentration of light (as derived in Abraham et al. 1994), mass-, and luminosity-weighted ages ${ }^{14}$ and the fractions of mass enclosed in the bulge (plots not shown). BSF early types are less concentrated and systematically younger than red early types. In addition, BSF early types and red early types of similar mass have a quite similar fraction of mass in the disk, but a few BSF galaxies host a higher fraction of mass in the disk than their red counterparts.

\subsection{Green Early Types}

Figure 9 shows the morphological distribution of green galaxies and blue and RP late types. Focusing on the first population, it emerges that it is mainly composed of late types, but it also includes some ellipticals and S0s. In Appendix B we will discuss in detail the differences between these two populations, but here we just focus on all early types.

The properties of green early types can be compared to those of green late types and BSF early types (Figure 7 and central panels of Figure 8). Green early types and BSF

\footnotetext{
14 Following the definition of Cid Fernandes et al. (2005), the luminosityweighted (mass-weighted) age is computed by weighting the age of each SSP composing the integrated spectrum with its bolometric flux (mass).
} 

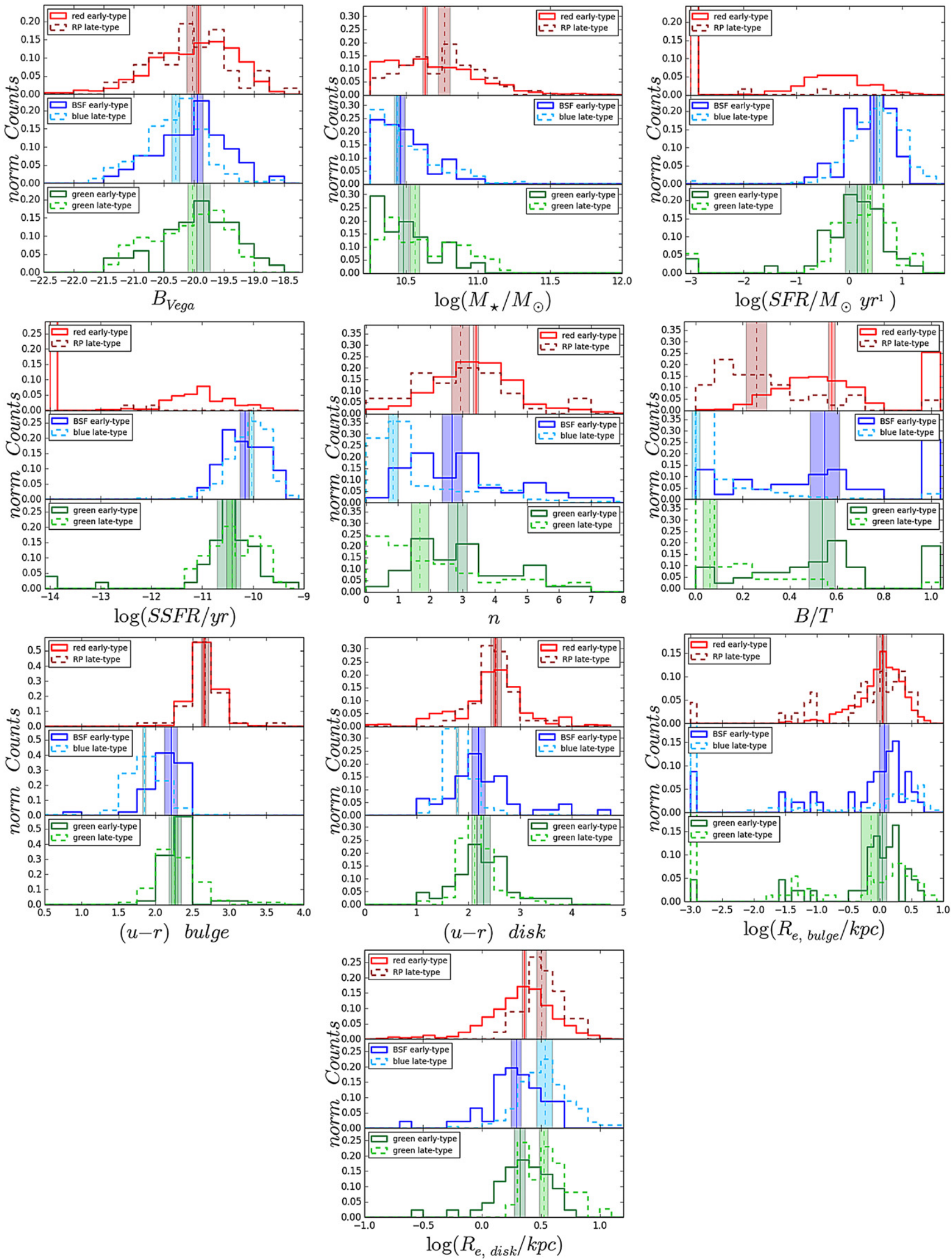

Figure 7. Normalized distributions for BSF early types (blue), green early types (dark green), red early types (red), blue late types (cyan), green late types (light green), and RP late types (dark red), as indicated in the labels. From top to bottom, left to right: $B_{\text {Vega }}$ magnitudes, stellar masses, SFRs, SSFRs, Sérsic indexes, B/T ratios, $(u-r)_{r f}$ of bulges, $(u-r)_{r f}$ of disks, bulge effective radii, and disk effective radii. Medians and errors on the medians are also shown. 

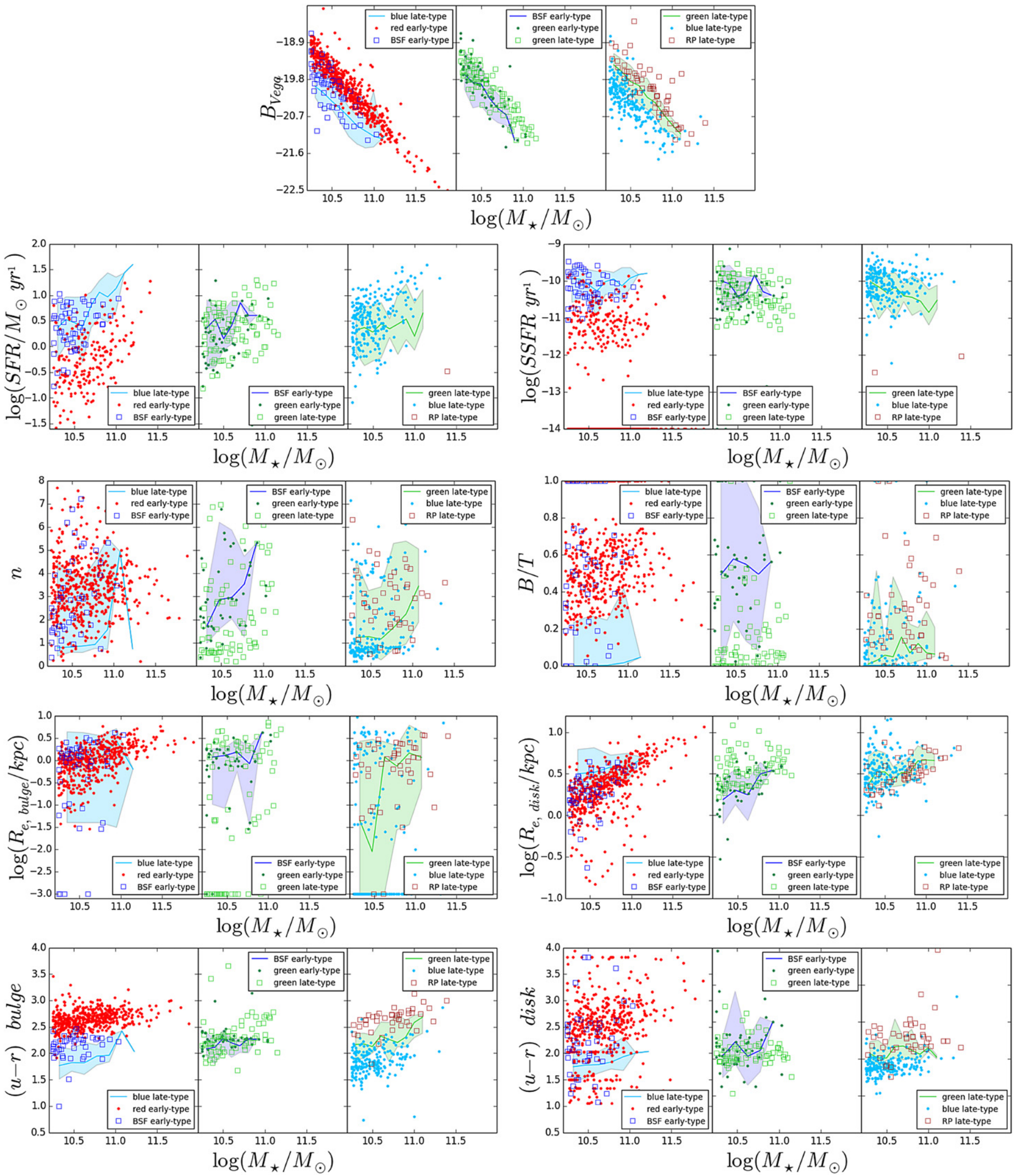

Figure 8. Properties as a function of mass for BSF early types (blue), green early types (dark green), red early types (red), blue late types (cyan), green late types (light green), and RP late types (dark red), as indicated in the labels. In each panel, three populations that might be evolutionarily linked are shown. For clarity, in each panel one population is represented by its median and a shaded area, including its 10th and 90 th percentiles. From top to bottom, left to right: $B_{\text {Vega }}$ magnitudes, SFRs, SSFRs, Sérsic indexes, B/T ratios, $(u-r)_{r f}$ of bulges, $(u-r)_{r f}$ of disks, bulge effective radii, and disk effective radii. 


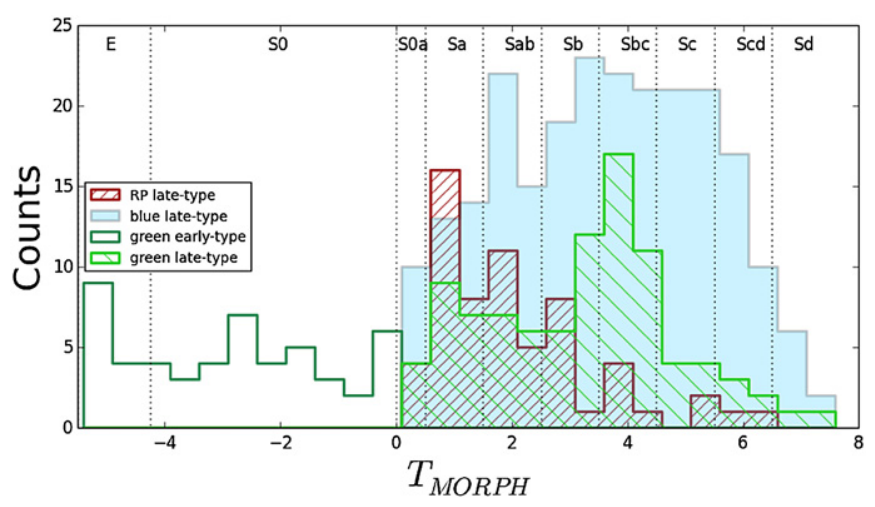

Figure 9. Finer morphological distribution of all (MMG+satellites) green, RP late types, and blue late types, above the mass completeness limit.

early types have similar mass distributions and maximum mass $\left(\log M_{\star} / M_{\odot} \sim 11.1\right)$, while green late types are slightly more massive. Green early types and BSF early types have similar Sérsic indexes, B/T ratios, similar colors in the bulge and the disk, and similar sizes of bulge and disk. At any fixed stellar mass, they only show a light reduction of star formation (SFR and SSFR).

\subsection{Green and Red Passive Late Types}

We now compare green, RP, and blue galaxies with a latetype morphology. Figure 9 shows that the RP morphological distribution is clearly skewed to earlier types compared to that of blue late types, but intermediate and late-type spirals are present too. Green galaxies show an intermediate distribution between the two.

Also, the mass distribution of green galaxies is intermediate between that of blue (less massive on average) and RP (more massive), even though the maximum mass reached is similar in the three samples (Figure 7).

In addition, most green late types are not yet completely quenched: their SSFR distribution indicates that most of them are still forming stars, just at a lower average rate than blue galaxies of similar mass. Only a few $(\sim 5 \%)$ green late types are already completely passive. The colors of both their bulges and disks are intermediate between red and blue, indicating that both of these structures are experiencing reduced levels of star formation (Figure 7 and right panels of Figure 8).

As for their structure, green late types are characterized by somewhat more prominent bulges than blue late types: on average, they have slightly larger Sérsic indexes, slightly higher $\mathrm{B} / \mathrm{T}$ ratios, and larger bulges than blue galaxies of similar mass.

Focusing on RPs, almost all of the distributions point to differences from blue galaxies: RP late-type galaxies are on average fainter in the $B$-band, much more massive, have higher $n$ and $\mathrm{B} / \mathrm{T}$, much redder bulges and disks, and quite similar bulge and disk sizes, but with the tendency to be on the smaller or more compact side of the size-mass distributions than blue late-type galaxies of similar mass.

In addition, at a given stellar mass, RP late types are also noticeably older and contain a higher fraction of mass in the bulge than blue late types (plots not shown).

\subsection{Poststarburst Galaxies}

Another way to understand how blue, star-forming galaxies turn into red, passive systems is to directly look at their spectra and look for signs of such a transition. Adopting the spectral classification defined by Dressler et al. (1999, MORPHS collaboration), we identify $k+a$ galaxies whose spectra display a combination of signatures typical of both K- and A-type stars with strong $\mathrm{H} \delta$ in absorption and no emission lines. These features are typical of so-called poststarburst or post-starforming galaxies whose star formation was recently (at some point during the last $0.51 \mathrm{Gyr}$ ) truncated over a short timescale, typically shorter than a few times $10^{8} \mathrm{yr}$.

In our mass-limited sample, $4.5 \%$ of all galaxies can be classified as $k+a$. This fraction is independent of environment. However, when considering the relative number of $k+a$ and blue galaxies, this is much higher in groups $(22 \% \pm 6 \%)$ than in binaries and singles $(13 \% \pm 5 \%)$, suggesting a higher efficiency of sudden quenching in groups compared to lower mass halos.

In our sample, $32 k+a$ are red (eight RP late types), 13 are green, and 10 are blue (one BSF early type). This shows that the majority of those galaxies that are truncated on a short timescale cannot be recognized based on color or color+morphology, but only by performing a detailed spectral analysis. The $k+a$ channel is therefore another transition channel that needs to be considered to build a complete picture of galaxy transformations and that affects $4.5 \%$ of all galaxies on a timescale of $\sim 1 \mathrm{Gyr}$.

Finally, we note that only $6 \%$ of green early types and $10 \%$ of green late types are $k+a$, confirming that most of the green galaxies are due to a star formation decline on a longer timescale.

\subsection{SFHs of the Different Populations}

We now investigate the SFHs of the different galaxy populations. To reduce the effect of the stellar mass in quenching galaxies, we consider two different mass bins: $10.25<\log M_{\star} / M_{\odot}<10.7$ and $10.7<\log M_{\star} / M_{\odot}<11.15$. We do not include more massive galaxies because of the lack of statistics. We verified that no significant residual dependence on mass distribution remains in each mass bin.

We compute the mean SFR per galaxy in five age intervals $(t=$ $0.877,4.079,9.021,11.863,12.345 \mathrm{Gyr}$ ) and compare the trends for galaxies of different colors or star-forming properties. Errors on mean values are obtained using a bootstrap resampling. Figure 10 shows that galaxies with different properties today are characterized by different average SFHs, which also depend on stellar masses.

Blue late types are currently forming stars, ${ }^{15}$ and the slope of their decline in star formation with time depends on stellar mass, being steeper for more massive galaxies. In both mass bins, green late-type galaxies have the same SFH as blue late types at early epochs, the only difference being a significant turndown of their star formation in the last $13 \mathrm{Gyr}$ (last one or two time bins depending on mass). This is consistent with green late types being regular blue late types until a few gigayears ago, when they experienced a reduction (but not yet a complete halt in most cases) of the star-formation activity. Green early and late types show very similar SFHs (plot not shown).

Except for the first bin at high masses, BSF early types have SFHs identical to blue late types. This is consistent with BSF early types being blue late types that suffered an alteration of their morphology. The average SFH of red early types with SSFR $<10^{-12} \mathrm{yr}^{-1}$ is instead very different from that of BSF early types and of all other subsamples: these galaxies were forming stars at a high rate at early epochs, and then their SFR

\footnotetext{
15 Blue late types with SSFR $<10^{-12} \mathrm{yr}^{-1}$ are $2 \%$ of all blue late types, and
} their influence is negligible. 


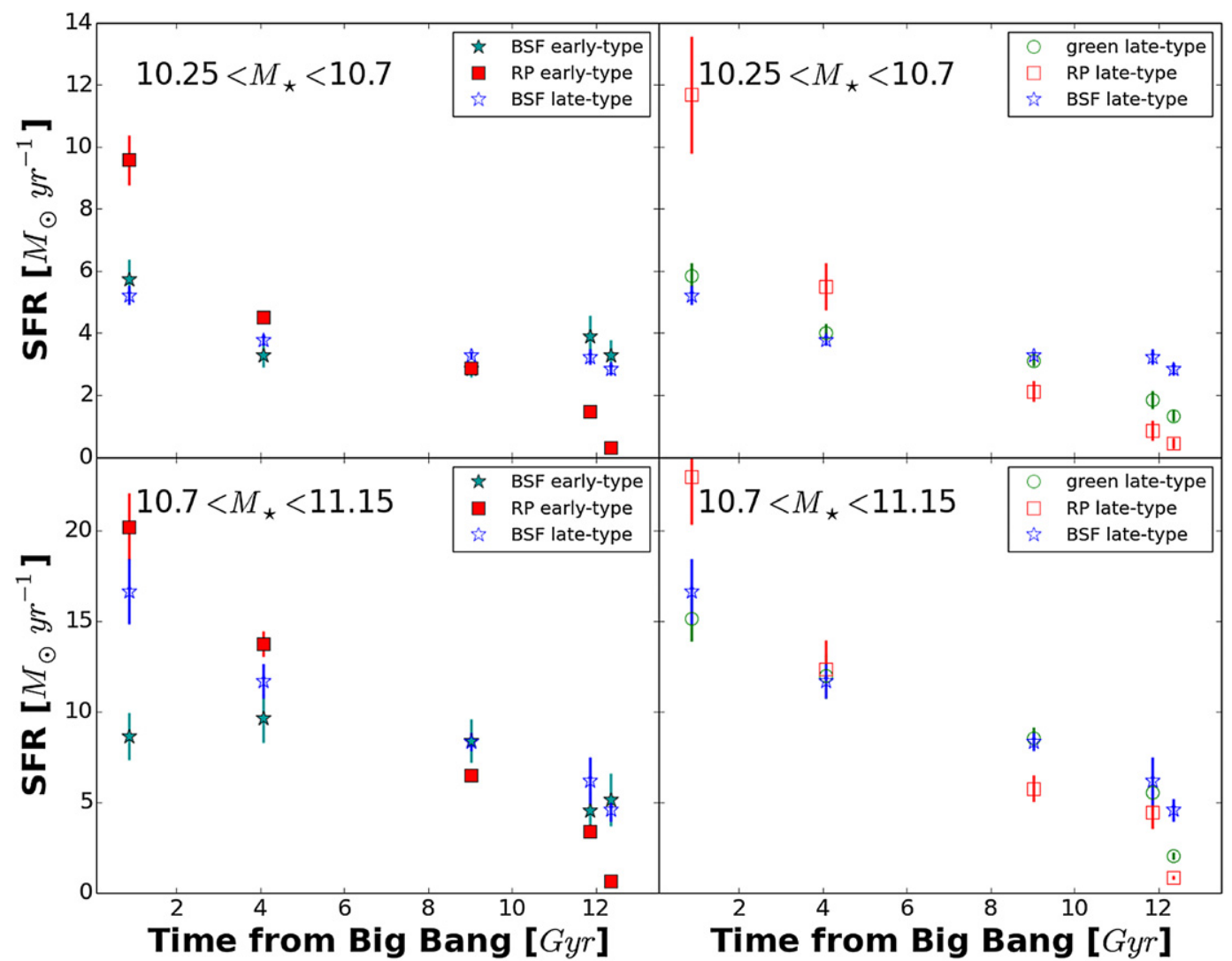

Figure 10. Mean SFR as a function of cosmic time for galaxies of different colors or star-forming properties, as described in the labels. Top panels: galaxies with $\log M_{\star} / M_{\odot}<10.7$. Bottom panels: galaxies with $10.7<\log M_{\star} / M_{\odot}<11.15$. Errors on mean values are obtained using a bootstrap resampling.

declined steeply with time. This effectively rules out the RP early types as candidate progenitors for the BSF early types.

Moving the attention to RP late types, their SFH strongly depends on stellar mass (being steeper at higher masses) and does not resemble that of blue late types, showing a much stronger variation with time. In the last $\sim 6$ Gyr their average SFR is lower than that of blue late types of similar mass. The RP late-type population most likely comprises all latetype galaxies that have stopped forming stars at any epoch and that have retained their morphology. Indeed, inspecting the SFH of individual galaxies, we find a number of RP late types that have stopped forming stars at intermediate or even high redshifts, $\sim 30 \%$ at $z \geqslant 0.3$. Therefore, it is quite logical to expect an average star-formation decline like the one observed: those late types that stopped forming stars at an early epoch only contribute in this plot in the oldest age bins, making the decline slope steepen. In this sense, it is not logical to search for an evolutionary direct connection between all of today's RP late types and today's blue late types: only the subset of latest arrivals of the former have recently evolved from the latter. The differences in the right panel of Figure 10 can be explained in such a scenario.

\section{COLOR EVOLUTION FOR DIFFERENT QUENCHING HISTORIES}

To investigate what type of SFH can produce a green galaxy spectrum and for how long, we employ our spectrophotometric model described in Section 2 (Fritz et al. 2007, 2011) to compute the color of galaxies with different histories and different quenching timescales. We use the SFH lognormal analytic form of Gladders et al. (2013):

$$
\operatorname{SFR}\left(t, t_{0}, \tau\right)=\frac{1}{t \sqrt{2 \pi \tau^{2}}} \exp -\frac{\left[\ln t-t_{0}\right]^{2}}{2 \tau^{2}}
$$

where $t$ is the elapsed time since the Big Bang, $t_{0}$ is the logarithmic delay time, and $\tau$ sets the rise and decay timescale. This form has been shown to be a good representation of galaxy SFHs.

We consider galaxies with various lognormal parameters $\left(t_{0}\right.$ and $\tau)$ and compute their $(U-B)$ rest-frame color assuming (1) no quenching at any time; (2) a sudden quenching, i.e., an abrupt interruption of the star-formation activity on a timescale $t_{\text {quench }}=0$; and (3) a linear decline of the SFR from the undisturbed level to zero with short to long timescales: $t_{\text {quench }}=$ $0.2,0.5,1,2,3$ Gyr. By construction, all of the histories have an SFR equal to zero 1.1 Gyr ago.

Figure 11 illustrates two emblematic cases of our modeling: one with a short $\tau$ (usually considered typical of early spirals) and one with a long $\tau$ (typical of late spirals). In the first case, galaxies can assume green colors for $\sim 2.5 \mathrm{Gyr}$ even without being subject to a quenching event, and in the second case intermediate colors are probably a sign of quenching. A short quenching time $\left(t_{\text {quench }}=0-0.5 \mathrm{Gyr}\right)$ produces a very short green phase $(\sim 0.15 \mathrm{Gyr})$, which might be hardly observable, and galaxies whose SFR declines with $t_{\text {quench }}=1-3$ Gyr go through a green phase that lasts for $0.250 .5 \mathrm{Gyr}$.

Galaxies with a very short $\tau$ ( $\sim 0.3$, typical of early types) do not go through a green phase, at least not in the last 6 Gyr (plot not shown). 

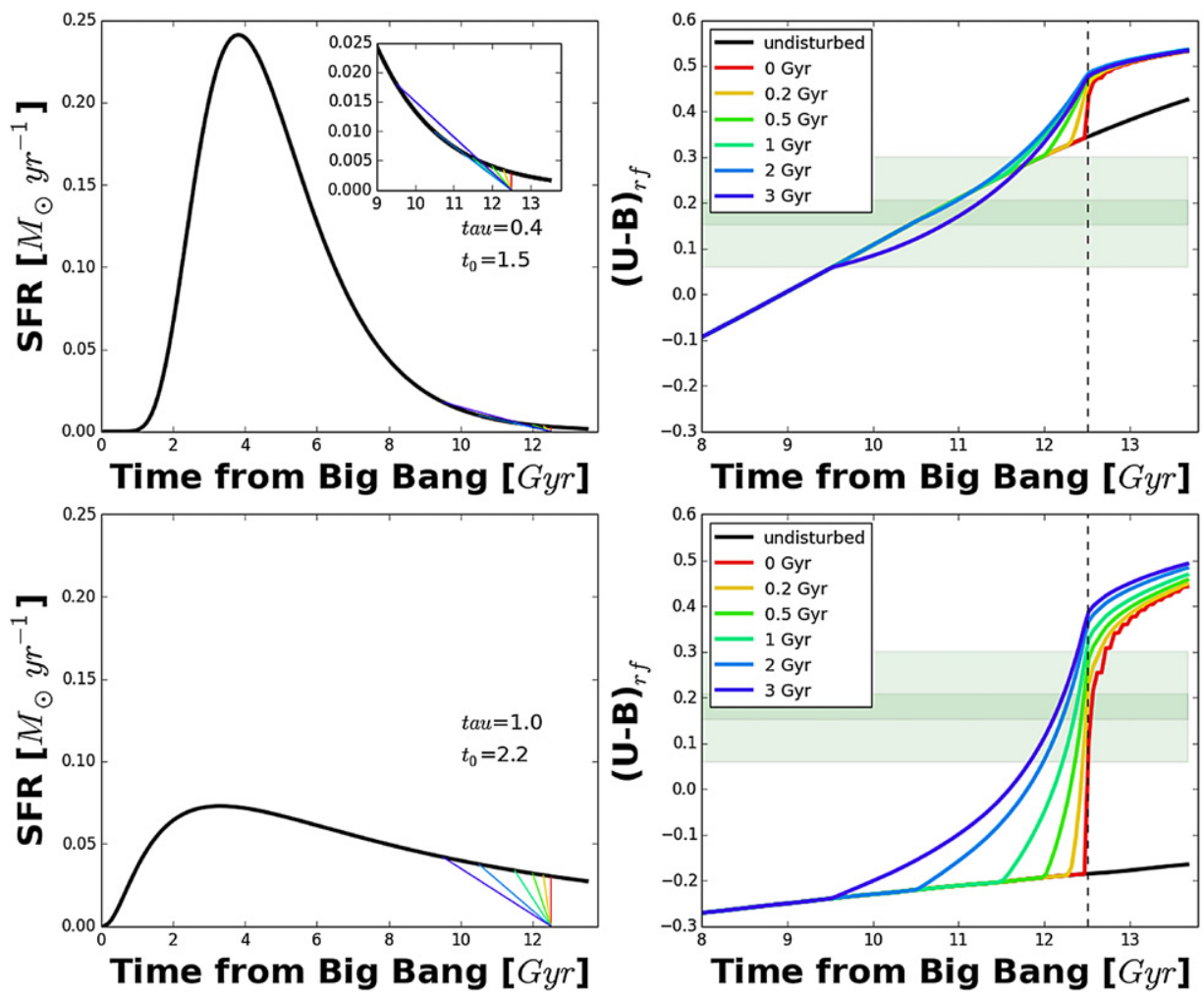

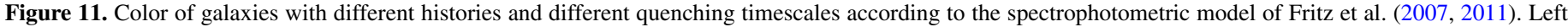

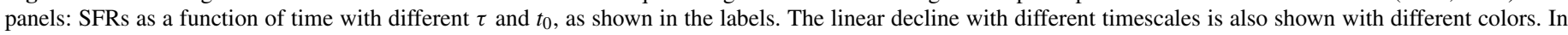

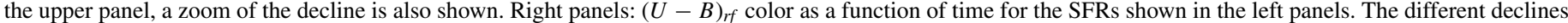
are also shown. Green shaded areas show our definition of green galaxies (see Section 3) for galaxies with $\log M_{\star} / M_{\odot}=10.25$ and $\log M_{\star} / M_{\odot}=11.5$.

To conclude, our modeling shows that green colors are due to SFHs declining with long timescales. These could be due either to "undisturbed" lognormal histories with short $\tau \mathrm{s}$, or to long$\tau$ galaxies that are quenched on the timescales on the order of $1 \mathrm{Gyr}$ or more. Green colors are therefore not necessarily indicative of "quenching" processes.

\section{DISCUSSION}

In this paper we have investigated how galaxies of different stellar mass transform from one type to another in a variety of environments (groups, binary systems, single galaxies) in the local universe. We found that a nonnegligible fraction of objects is likely in a transitional phase, that is, they are experiencing or have recently experienced a transformation from being starforming to passive, or vice versa. We have seen that there is not a one-to-one correspondence between color and morphology, and we confirmed that in many cases these two quantities must change on different timescales and might have a different dependence on stellar mass, environment, and galaxy class. We have then investigated the properties of the galaxies in transition and compared them to those of their "normal" counterparts, to understand whether their structural properties are also different, or whether variations are limited to the efficiency of the SFR, which is mainly reflected in the galaxy color. Finally, we inspected SFHs to gain insights into the rate at which all galaxies have been forming stars in the past.

\subsection{A Possible Evolutionary Scenario}

We are now in the position of proposing a possible evolutionary scenario for galaxies, as summarized in Figure 12.

From blue late types...
We start considering blue (star-forming) late-type galaxies. At a certain epoch, because of secular and/or environmental processes, their gas supply is affected and their SFR starts to decline, either on a short or long timescale. Therefore, a change in color must occur, either accompanied by a change in morphology or not. Various scenarios are possible at this stage.

\section{...to green late types}

If the SFR declines, blue late types can turn into green late types. Indeed, these two families share a similar SFH for most of the time, with green late types showing a reduction of the SFR only in the last few gigayears (Figure 10). Therefore, green late types are most likely the result of blue late types that have had their SFR suppressed, but not yet extinguished, both in the bulge and in the disk. The analysis of their structural parameters is consistent with this picture if a small structural variation takes place, in the sense of a small increase in the bulge relevance from blue to green. According to our modeling, galaxies can assume green colors for $\sim 2.5 \mathrm{Gyr}$ if their SFR is characterized by a short $\tau$, even without being subject to any quenching event. Alternatively, galaxies whose SFR is characterized by a long $\tau$ can remain green for up to $1 \mathrm{Gyr}$ as a consequence of a quenching event.

... or to BSF early types

If the morphology changes before star formation is shut off, blue late types can turn into BSF early types. The analysis of the SFHs and their mass distributions support this hypothesis. Structural parameters of blue late types and BSF early types show significant differences instead, as expected if a morphological transformation has occurred. At any given mass, the disk in BSF early types is not only smaller and fainter (different $\mathrm{B} / \mathrm{T}$ ratio distribution) but also less massive, suggesting that the 


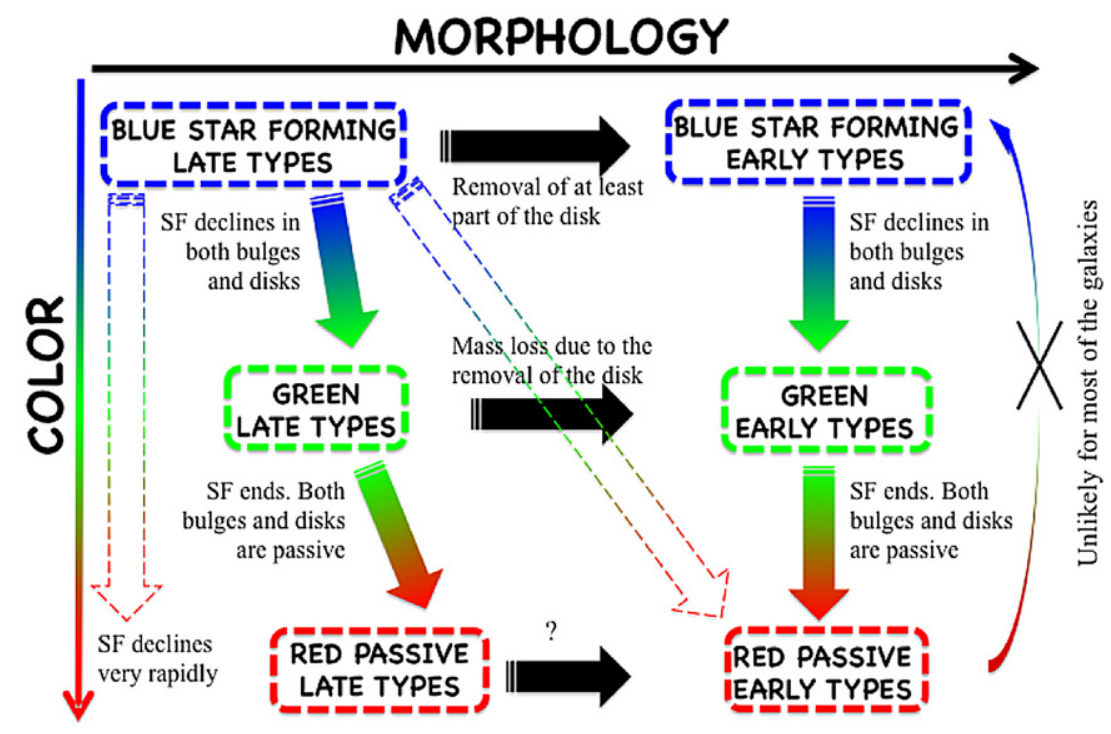

Figure 12. Illustration of our main results and interpretation.

morphological transformation happened with a removal of at least part of the stellar disk.

From green late types...

Subsequently, green late types can further undergo a change in color and turn into RP late types, or change their morphology and become green early types.

... to RP late types

If no major morphological transformation occurs, ${ }^{16}$ green late types most likely become RP late types. Compared to their first progenitors (blue late types) and their possible recent progenitors (green late types), RP late types are systematically more massive and at a given stellar mass have a smaller disk. These results, together with the analysis of SFHs, suggest that RP late types today cannot derive only from the current blue and green late types via the quenching and fading of a disk. They are probably a heterogeneous population that comprises all latetype galaxies that have stopped forming stars at any epoch and that retained their morphology. The nonnegligible fraction of poststarburst galaxies $(\sim 15 \%)$ in RP late types suggests that some of these objects became red after a very short (therefore virtually unobservable) green phase.

\section{... or to green early types}

If green late types are subject to a morphological transformation, they might become green early types. The analysis of the SFH supports such a possibility because no differences in SFH have been detected between the two populations (plot not shown). Green early types are characterized by a steeper mass distribution than the green late types, which extends toward slightly higher masses. The analysis of the structural parameters suggests that this mass loss is mainly related to the progressive disappearance of the disk, which at any given stellar mass is smaller and less massive. The properties of the bulges of green late types and green early types are more similar; as a consequence, the relative proportion of bulges and disk changes, as reflected in the distribution of $\mathrm{B} / \mathrm{T}$ ratios.

From BSF early types to green early types

Green early types can also be derived from BSF early types that suffer a reduction of their SFR and a consequent change in

\footnotetext{
16 A minor morphological transformation from late spirals to slightly earlier spirals is favored by the morphological distributions of green late types versus blue late types.
}

color. Indeed, green and BSF early types present very similar mass and structural parameter (Sérsic indexes, B/T ratios, size of bulges and disks) distributions, which supports this scenario.

From green early types and RP late types to red early types

Finally, both green early types and RP late types can eventually turn into red early types. However, given the fact that the RP early-type population contains galaxies that stopped forming stars at any earlier epoch, which were characterized by different structural properties, comparisons between such populations are very hard, and it is difficult to state the frequency of such transformations.

In principle, red early types might suffer a rejuvenation process that makes them change colors. Indeed, some structural parameters of red and BSF early types are similar, consistent with the hypothesis of a common origin. However, this scenario seems to be ruled out by the characterization of the SFHs that are clearly different for the two populations.

Adopting a different galaxy selection, Tojeiro et al. (2013) found that red late-type spirals are recent descendants of blue late-type spirals, sharing similar SFHs at early times and showing a reduction of the star formation only in the last $500 \mathrm{Myr}$. On the basis of the SFH and dust content, they claimed that red early-type spirals are more likely to evolve directly into red ellipticals than are red late-type spirals. They also found that blue ellipticals show SFHs similar to blue spirals, except for a reduction of the efficiency of star formation in the last 100 Myr. Blue ellipticals have a different dust content than all spiral galaxies, ruling out the scenario in which most of them are derived from blue spirals.

\subsection{Physical Processes Responsible for Galaxy Transformations}

Different mechanisms have to be at work to produce the observed transformations.

Color transformations are due to a reduction and suppression of the SFR both in bulges and disks. They can occur as the result of an external process or simply because galaxies use up all of their gas and shut down their star formation while they retain their structure.

In the case of late types, by taking realistic accretion histories from cosmological simulations, Forbes et al. (2012) have shown 
that a certain fraction of disks in the course of their lifetimes is expected to experience a period of low accretion, during which they will exhaust their gas supply and become redder, only to return to the blue cloud with the resumption of higher accretion rates.

Ram pressure stripping of cold gas (Gunn \& Gott 1972; Feldmann et al. 2011) and strangulation of the galactic system by removal of hot and warm gas necessary to fuel star formation (Larson et al. 1980; Balogh et al. 2000a; Font et al. 2008) might be responsible for such transformations, because they are expected to not affect galaxy morphology, at least not directly. However, we do not detect any environmental dependence (see Section 7.3), suggesting that these processes are not very efficient. In addition, while ram pressure is observed to act on galaxies in high-mass clusters, it has not been observed in lower mass groups, where lower halo gas densities and satellite velocities likely lower its efficiency. Moreover, halos of $M_{*} \sim 10^{12} M_{\odot}$ are not expected to have virial shock fronts that support hot, virialized gas within the halo (Dekel \& Birnboim 2006), so in this mass regime it is not clear that either strangulation or ram pressure can be efficient. This may suggest that tidal stripping (e.g., Park et al. 2007 and references therein), harassment, or mergers induce a rapid cold gas consumption that quenches star formation. However, all of these processes are also responsible for a morphological variation because the distribution of the light and gas are also affected. Because the external parts of the galaxies first feel these processes, it is plausible that they first affect disks, producing their observed fading. Therefore, a morphological change in the direction of an increase of the $\mathrm{B} / \mathrm{T}$ ratio might actually be an aftermath of quenching, simply due to the fading and the reduction of a star-forming disk once star formation is reduced or ceased.

\subsection{Lack of Environmental Effects}

Our analysis has revealed very little evidence for environmental effects on galaxy transformations.

The relative proportion of green and blue galaxies has been found to be almost constant with environment (groups, binaries, satellites, and as a function of group-centric radius) and also for MMGs and satellites, implying that changes in color occur on a timescale that does not depend on host halo mass or halo-centric radius or on being a central or a satellite in a halo. Alternatively, if most green galaxies are not due to quenching, the constancy of the green-to-blue ratio would indicate that the processes giving origin to long- and short- $\tau$ SFHs do not change efficiency with environment. The decline in star formation giving rise to green galaxies is therefore not due to environmental effects. On the other hand, the relative fraction of green and blue galaxies depends on mass, being a factor 2:1 at high masses, 1:1 at intermediate masses, and $\sim 1: 3$ at lower masses, suggesting that the decline in star formation in green galaxies is related to the galaxy mass.

The only detected environmental effects are that (1) in single MMGs, at any given mass, there are proportionally more blue and green galaxies than in the other environments, and in general they are proportionally less massive and (2) in groups there might be a higher fast quenching efficiency that gives origin to poststarburst signatures.

Our findings might be quite surprising, given that a host halo's virial radius broadly corresponds to a physical transition from the low-density field environment to a high-density region where dark matter and gas are virialized, so environmental effects would be expected.
However, it is important to keep in mind that environmental dependences can extend to galaxies beyond the virial radius of a group or cluster (as largely discussed in Wetzel et al. 2014). Therefore the (lack of) trends are plausibly driven, at least in part, by those galaxies having passed within much smaller distances from a group or cluster, but at the time of the observations they are found very far from it. Also, a large fraction of central galaxies near massive host halos are actually ejected satellites (e.g., Wetzel et al. 2014). Balogh et al. (2000b) first noted from N-body simulations of clusters that particles that have passed within the virial radius can then orbit well outside of it. Ejected (or backsplash) satellites (see, e.g, Gill et al. 2005; Bahé et al. 2013) typically orbit back out to a maximum distance of $\sim 2.5 R_{200}$ beyond a host halo after passing through it. In addition, almost half of all galaxies within this distance are composed of these ejected satellites, which are preferentially quiescent (e.g., Wang et al. 2009; Wetzel et al. 2013), with higher fractions for less massive galaxies and around more massive host halos. Thus, ejected satellites are potentially critical for understanding the properties of galaxies near groups or clusters and obtaining a complete picture of environmental dependence.

\subsection{The Importance of the Galaxy Structure}

Recently, the importance of galaxy structure for galaxy transformations has been widely discussed in the literature.

Carollo et al. (2014) report that the quenched satellites at low $z$ have larger B/T and smaller half-light radii than the starforming satellites. They find that differences are mostly due to differences in the disks, which have lower luminosities in the quenched galaxies, but they cannot be explained by uniformly fading the disks following quenching. Instead, either there must be a differential fading of the disks with galaxy radius or disks were generally smaller in the past, both of which would be expected in an inside-out disk growth scenario.

Other literature results focused mainly on the bulge, finding that its properties might play a role in quenching galaxies, rather than the disk. For example, in the local universe, Abramson et al. (2014) showed that the increase in bulge mass fractions, which are portions of a galaxy not forming stars, is responsible for the existing anticorrelation between SSFR and $M_{*}$. At $z<0.2$, the passive fraction for central galaxies has been found to be closely correlated to the bulge mass (Bluck et al. 2014) and to the B/T ratio (Omand et al. 2014). At $0.5<z<2.5$, Lang et al. (2014) found an increased bulge prominence among quiescent galaxies, with an increase of the typical B/T among star-forming galaxies above $10^{11} M_{\odot}$. These findings have led some authors to suggest that the physical mechanisms responsible for the quenching of star formation must be strongly coupled to the formation or accretion of the bulge.

Our analysis confirms that the relative importance of the bulge and the disk appears to be a key parameter in galaxy transformations. We found that a morphological transition (from $\mathrm{BSF}$ late types to BSF early types, or from green late types to green early types) is mainly due to the fading and total or partial removal of the disk. A transition in star formation with no morphological change (from blue late types to green late types) instead is accompanied by a small structural change: as we have seen in Section 5.3, the average $\mathrm{B} / \mathrm{T}$ ratio and mass ratio of bulge and disk in green late types is only slightly higher than in blue late types. In this case, the differences in these ratios can be ascribed to bulge growth instead of disk fading, but the effect is overall small on the population. 


\subsection{Quenching Times}

According to our modeling, galaxies that sustain a green color for a nonnegligible interval of time $(>0.5 \mathrm{Gyr})$ can originate either from a long timescale $(>1 \mathrm{Gyr})$ quenching of long$\tau$ galaxies, or from short- $\tau$ "undisturbed" SFHs typical of intermediate types. Galaxies with a short quenching (00.5 Gyr) timescale do exist, but they sustain green colors for a short period of time, and they are observable as $k+a$ galaxies that quickly transition from being blue to being red.

We have shown that the occurrence of green galaxies (compared to that of blue galaxies) does not depend on environment, but that of $k+a$ does. This suggests that the environmental quenching timescale is short, while other galaxies go from being star-forming to being passive on a long timescale independently of environment.

We have seen that green late types are twice as numerous as $k+a$ 's. Starting from the logical assumption that both green and $k+a$ galaxies have a common progenitor among star-forming galaxies, and if we assume that the green phase lasts for about twice the time (on the order of 2 Gyr) of the $k+a$ visibility ( $\sim 1 \mathrm{Gyr})$, we conclude that the short timescale and the long timescale star-formation "quenching" channels contribute about equally to the growth of the passive population.

Several authors have tried to estimate the "quenching timescale" using different approaches. A direct comparison is impossible because it strongly depends on how a "quenching or quenched" galaxy is defined. However, we report here some of the latest results for comparison.

Wetzel et al. (2013) found that satellite quenching is the dominant process for building up all quiescent galaxies at $M_{*}<$ $10^{10} M_{\odot}$. They proposed a "delayed then rapid" quenching scenario: satellite SFRs are unaffected for 24 Gyr after infall, after which star formation quenches rapidly, with an e-folding time of $<0.8$ Gyr (see also McGee et al. 2009, 2011; De Lucia et al. 2012). These quenching timescales are shorter for more massive satellites but do not depend on host halo mass.

Investigating objects in transition defined using a color-color diagram, Mok et al. (2013) proposed a much shorter quenching timescale with an $e$-folding time of $<0.5$ Gyr. Schawinski et al. (2014), inspecting morphologies in addition to colors, concluded that only a small population of blue early types move rapidly across the green valley after the morphologies are transformed from disk to spheroid and star formation is quenched rapidly ( $\tau<0.25 \mathrm{Gyr}$ ). In contrast, the majority of BSF galaxies have significant disks, and they retain their latetype morphologies as their star-formation rates decline very slowly ( $\tau>1$ Gyr).

Only Wheeler et al. (2014), studying a sample of dwarf galaxies, found much longer timescales $(>9.5 \mathrm{Gyr}$, a "slow starvation" scenario), concluding that the environmental processes triggering quenching must be highly inefficient.

\section{SUMMARY AND CONCLUSIONS}

Investigating a mass-complete sample of galaxies drawn from the PM2GC (Calvi et al. 2011), this paper focused mainly on two points: (1) characterizing the color (red, green, blue) and morphological (ellipticals, S0s, late types) transformation of galaxies as a function of the stellar mass and the environment and (2) studying the properties of the objects that are most likely in a transitional phase (green, RP late types, BSF early types), with the aim of understanding the evolutionary links between the different subpopulations.
Our analysis showed that the relative importance of the bulge and the disk seems to play an important role in galaxy transformations. We found that the fading and total or partial removal of the disk produces a morphological transition, such that BSF late types and green late types become BSF early types and green early types, respectively. On the other hand, a transition can occur even without a noticeable structural and morphological change: the SFR can decline both in bulges and disks, producing a variation in color. In this way, blue galaxies turn into green (when they are still forming stars, but a reduced rate) and red galaxies. Therefore, RP late-type galaxies descend from blue late types that have stopped forming stars at any epoch (retaining their morphology), going through either a short or long green phase.

Our spectrophotometric model allowed us to better characterize the occurrence and duration of the green phase. In some cases, galaxies can turn from blue to red quite quickly, going through a very short green phase $(<0.1 \mathrm{Gyr})$ that is hardly observable when considering only colors, but recognizable by their spectral $k+a$ features. In other cases, green colors are not indicative of "quenching" processes. They are due to SFHs declining with long timescales. These could be due either to "undisturbed" lognormal histories with $\tau$ typical of early spirals or to a long $\tau$ typical of late spirals that are quenched on the timescales on the order of at least $1 \mathrm{Gyr}$.

We thank the anonymous referee whose comments helped to improve the readability of the paper. This work was supported by the World Premier International Research Center Initiative (WPI), MEXT, Japan. It was also supported by the Kakenhi Grant-in-Aid for Young Scientists (B)(26870140) from the Japan Society for the Promotion of Science (JSPS). We thank Joe Liske, Simon Driver, and the whole MGC team for making their valuable data set public and easily accessible.

\section{APPENDIX A}

\section{TRENDS WITH SÉRSIC INDEXES}

In this paper we have investigated the relationship between color and morphology. In this appendix we show that adopting the Sérsic index instead of morphology to distinguish between the subpopulations overall gives similar results, even though the analysis of the morphologies is more detailed.

We separate the sample into bulge-dominated galaxies $(n>$ 2.5 , hereafter simply "bulges") and disk-dominated galaxies $(n<2.5$, hereafter simply "disks"). Figure 13 shows the same as Figure 2. Bulges are most likely red and more massive, while disks tend to be bluer. As shown in Table 3, bulge and disk fractions depend on the environment: for both MMGs and satellites, bulges are more common in groups than in single systems. However, in a given environment, fractions differ for satellites and MMGs only in groups, with MMGs hosting a higher fraction $(\sim 70 \%)$ of bulges than satellites $(\sim 60 \%)$. For both MMGs and satellites and in all environments, the distribution of disks resembles the one presented for late types, and the distribution of bulges resembles that of the sum of ellipticals and SOs.

Figure 14 shows the same as Figure 3. In both MMGs and satellites, blue and green disks show trends similar to blue and green late types, that is, they are more frequent at low masses than at higher. In MMGs, together they dominate the total population at low masses, while in satellites red bulges always dominate, with their importance increasing with increasing 

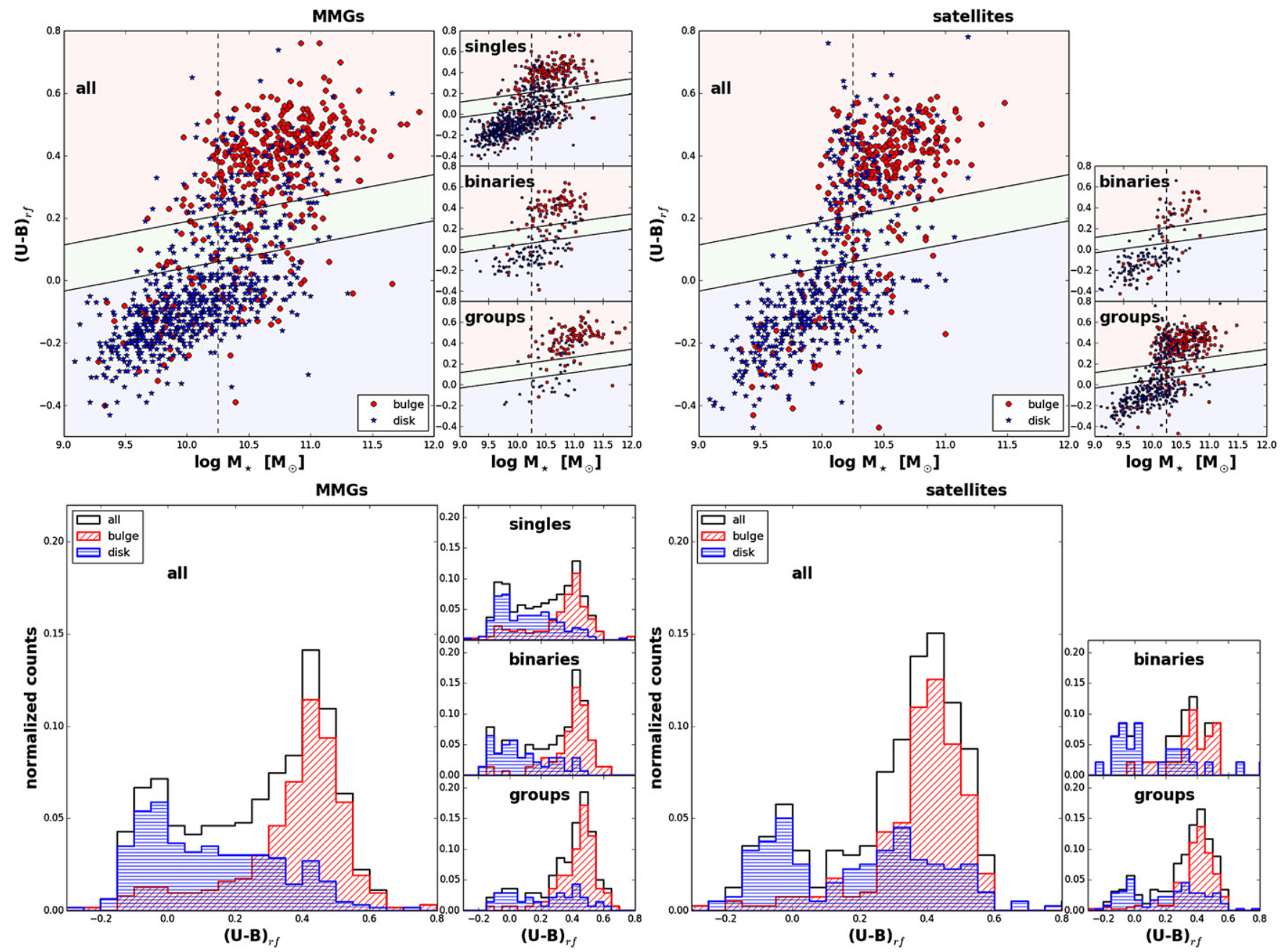

Figure 13. Rest-frame $(U-B)_{r f}$-mass relation (upper panels) and rest-frame $(U-B)_{r f}$ color distribution (bottom panels) for galaxies of different types (left panels: MMGs; right panels: satellites) and Sérsic indexes in the different environments (shown in the smaller windows). Red lines and circles: bulges; blue lines and stars: disks. In the upper panels, the black dashed vertical line represents the mass completeness limit, and the black solid line shows the separation between red, green, and blue galaxies.

Table 3

Percentage of Galaxies of Different Types Above the Stellar Mass Completeness Limit in Different Environments

\begin{tabular}{|c|c|c|c|c|c|c|c|c|c|}
\hline & & \multicolumn{2}{|c|}{ All Galaxies } & \multicolumn{2}{|c|}{ Single Galaxies } & \multicolumn{2}{|c|}{ Binary Systems } & \multicolumn{2}{|c|}{ Groups } \\
\hline & & Bulge & Disk & Bulge & Disk & Bulge & Disk & Bulge & Disk \\
\hline \multicolumn{10}{|c|}{ Most Massive Galaxies } \\
\hline \multirow{4}{*}{ Color } & All & $54 \pm 3$ & $46 \pm 3$ & $49 \pm 4$ & $51 \pm 4$ & $56 \pm 6$ & $44 \pm 6$ & $67 \pm 6$ & $33 \pm 6$ \\
\hline & Red & $45 \pm 3$ & $11 \pm 2$ & $37 \pm 4$ & $15 \pm 3$ & $48 \pm 6$ & $10 \pm 4$ & $62 \pm 6$ & $17 \pm 5$ \\
\hline & Green & $3 \pm 1$ & $9 \pm 2$ & $3 \pm 1$ & $12 \pm 2$ & $5 \pm 3$ & $8 \pm 4$ & $3 \pm 2$ & $4 \pm 3$ \\
\hline & Blue & $5 \pm 1$ & $21 \pm 2$ & $8 \pm 2$ & $23 \pm 3$ & $2 \pm 2$ & $26 \pm 5$ & $2 \pm 2$ & $11 \pm 4$ \\
\hline \multicolumn{10}{|c|}{ Satellites } \\
\hline \multirow{4}{*}{ Color } & All & $57 \pm 3$ & $43 \pm 3$ & $\ldots$ & $\ldots$ & $47 \pm 11$ & $53 \pm 11$ & $59 \pm 4$ & $42 \pm 4$ \\
\hline & Red & $51 \pm 4$ & $23 \pm 3$ & $\ldots$ & $\ldots$ & $40 \pm 11$ & $19 \pm 9$ & $52 \pm 4$ & $22 \pm 3$ \\
\hline & Green & $3 \pm 1$ & $5 \pm 2$ & $\ldots$ & $\ldots$ & $4 \pm 5$ & $4 \pm 5$ & $3 \pm 1$ & $5 \pm 2$ \\
\hline & Blue & $3 \pm 1$ & $16 \pm 2$ & $\ldots$ & $\ldots$ & $2 \pm 4$ & $30 \pm 10$ & $3 \pm 1$ & $14 \pm 3$ \\
\hline
\end{tabular}

mass. In general, red bulges resemble red early types. We note that in satellites the contribution of red bulges increases from $40 \%$ to $100 \%$ without showing any dip. This might suggest that the weird trend seen in red early and late types is due to galaxies that are morphologically classified as late but have a nonnegligible bulge. In both classes, trends for BSF bulges, RP disks, and green bulges are almost flat. All of these galaxies are absent only in the highest mass bin. Each subpopulation represents $\leqslant 15 \%$ of the total population.

Focusing on groups, Figure 15 shows the same as Figure 6. Both at low and high masses, within the errors, trends for satellites and MMGs are compatible. At low masses, the 


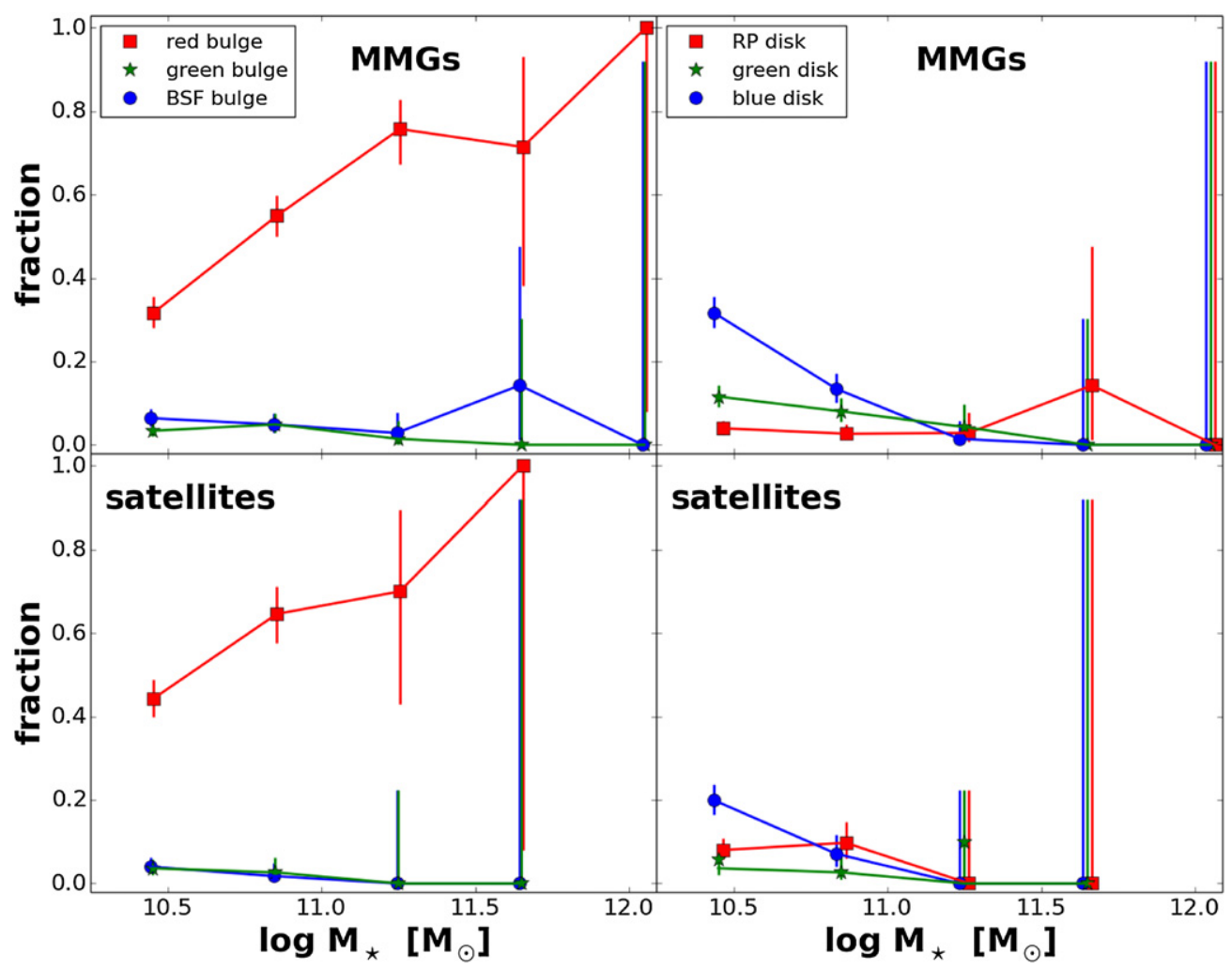

Figure 14. Fraction of galaxies as a function of stellar mass for MMGs (upper panels) and satellites (bottom panels), when a color+SSFR+Sérsic index cut is adopted. Left panels: bulges, as indicated in the labels. Right panels: disks, as indicated in the labels. Errors are defined as binomial errors (Gehrels 1986).
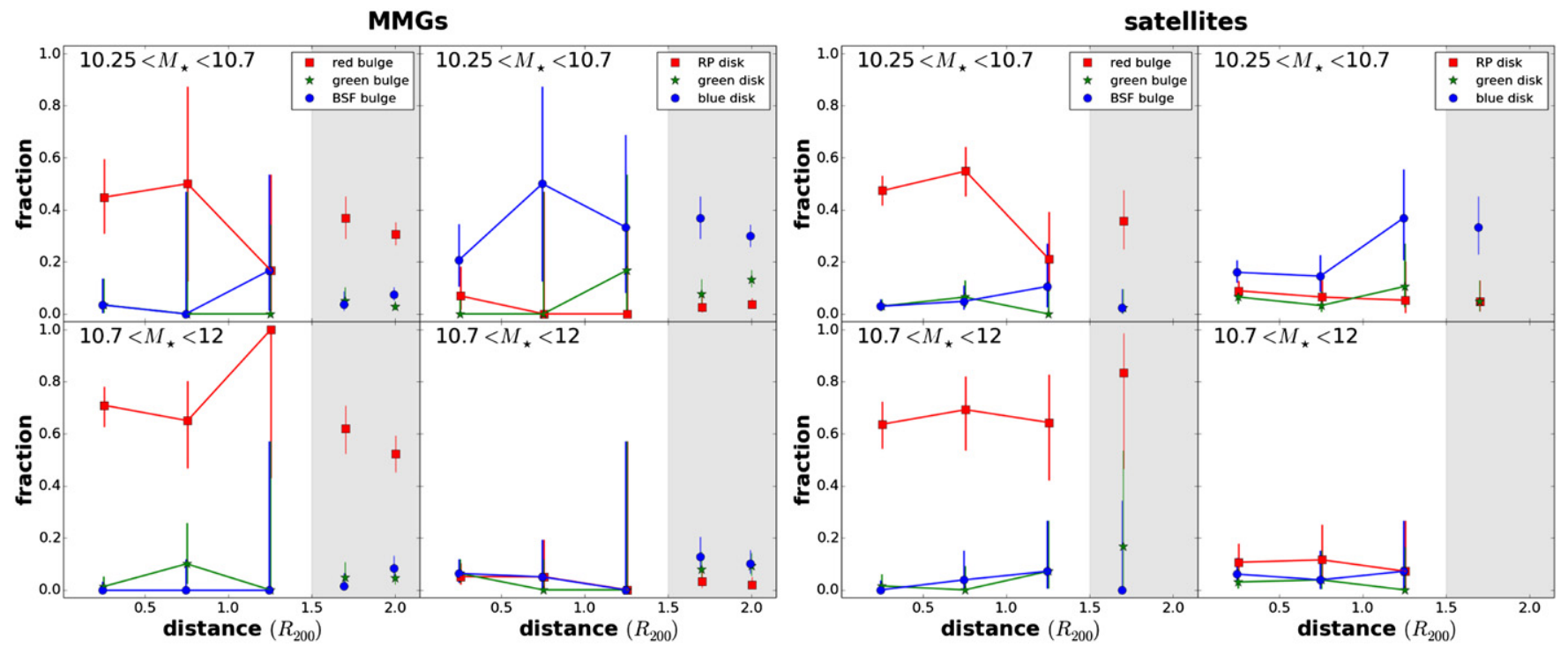

Figure 15. Fraction of galaxies as a function of group-centric distances in bins of stellar mass, when a color+SSFR+Sérsic index cut is adopted. Left panel: MMGs. Right panel: satellites. Upper panels: $\log M_{\star} / M_{\odot}<10.7$; lower panels: $\log M_{\star} / M_{\odot}>10.7$. Colors and symbols are as in Figure 14. Gray areas represent galaxies that are not in groups, i.e., binary systems (placed at $r / R_{200}=1.7$ ) and single galaxies (placed at $r / R_{200}=2$ ). Errors are defined as binomial errors (Gehrels 1986 ).

incidence of red bulges (which dominate close to the group centers) seems to decrease at large distances. In contrast, the fraction of blue and green disks and BSF disks slightly increases. Green bulges are almost absent at all distances. At higher masses, both in MMGs and satellites, red bulges dominate at all distances $(>60 \%)$, and their fraction increases with distance. In contrast, the other three classes of objects show slightly declining trends. In the nongroup environments, in both mass bins the fractions are similar to those of group outskirts, given the large uncertainties.

\section{APPENDIX B}

\section{ELLIPTICALS AND SOS}

In the main text we have presented results for ellipticals and S0s together, even though it is known that they are characterized by different properties. Table 4 presents the characteristic numbers for the two populations.

Red ellipticals and S0s differ especially in the B/T ratio, with the former being more bulge dominated than the latter. Similar discrepancies are also found for the objects in transitions. In 
Table 4

Characteristic Numbers of Ellipticals and S0s, Compared to Normal Galaxies of the Same Morphology

\begin{tabular}{|c|c|c|c|c|c|c|}
\hline \multirow[t]{2}{*}{ Quantity } & \multicolumn{3}{|c|}{ Ellitpicals } & \multicolumn{3}{|c|}{ S0s } \\
\hline & Red & Green & BSF & Red & Green & BSF \\
\hline Number & 295 & 15 & 18 & 281 & 36 & 32 \\
\hline$\left\langle B_{\text {Vega }}\right\rangle$ & $-19.97 \pm 0.05$ & $-19.8 \pm 0.2$ & $-20.4 \pm 0.1$ & $-19.87 \pm 0.05$ & $-19.8 \pm 0.1$ & $-20.28 \pm 0.09$ \\
\hline$\left\langle\log M_{\star} / M_{\odot}\right\rangle$ & $10.66 \pm 0.05$ & $10.4 \pm 0.1$ & $10.40 \pm 0.06$ & $10.62 \pm 0.05$ & $10.48 \pm 0.06$ & $10.39 \pm 0.07$ \\
\hline$\langle\mathrm{SFR}\rangle\left(M_{\odot} \mathrm{yr}^{-1}\right)$ & $0 \pm 0$ & $1 \pm 1$ & $4.7 \pm 0.7$ & $0 \pm 0$ & $1.8 \pm 0.5$ & $3 \pm 1$ \\
\hline$\langle\mathrm{SSFR}\rangle^{\mathrm{a}}\left(\mathrm{yr}^{-1}\right)$ & $(8 \pm 5) 10^{-12}$ & $(3 \pm 3) 10^{-11}$ & $(1.5 \pm 0.2) 10^{-10}$ & $(9 \pm 3) 10^{-12}$ & $(4 \pm 1) 10^{-11}$ & $(7 \pm 3) 10^{-11}$ \\
\hline$\langle n\rangle$ & $3.4 \pm 0.1$ & $2.8 \pm 0.4$ & $2.3 \pm 0.5$ & $3.3 \pm 0.1$ & $2.7 \pm 0.4$ & $1.8 \pm 0.4$ \\
\hline$\langle\mathrm{B} / \mathrm{T}\rangle$ & $0.61 \pm 0.02$ & $0.58 \pm 0.08$ & $0.7 \pm 0.1$ & $0.51 \pm 0.02$ & $0.52 \pm 0.06$ & $0.25 \pm 0.08$ \\
\hline$\left\langle R_{e}\right.$ (bulge) $\rangle(\mathrm{kpc})$ & $1.13 \pm 0.07$ & $0.9 \pm 0.2$ & $1.2 \pm 0.3$ & $1.09 \pm 0.08$ & $1.2 \pm 0.3$ & $1.2 \pm 0.2$ \\
\hline$\left\langle R_{e}(\right.$ disk $\left.)\right\rangle(\mathrm{kpc})$ & $2.4 \pm 0.1$ & $2.2 \pm 0.4$ & $1.5 \pm 0.2$ & $2.1 \pm 0.1$ & $2.0 \pm 0.2$ & $2.0 \pm 0.2$ \\
\hline$\langle(u-r) \text { bulge }\rangle^{\mathrm{b}}$ & $2.66 \pm 0.04$ & $2.2 \pm 0.1$ & $1.95 \pm 0.09$ & $2.65 \pm 0.01$ & $2.3 \pm 0.1$ & $1.91 \pm 0.06$ \\
\hline$\langle(u-r) \operatorname{disk}\rangle^{\mathrm{b}}$ & $2.52 \pm 0.05$ & $2.2 \pm 0.3$ & $2.0 \pm 0.3$ & $2.53 \pm 0.06$ & $2.3 \pm 0.1$ & $1.9 \pm 0.1$ \\
\hline
\end{tabular}

Notes.

${ }^{a}$ Values computed only with galaxies with $\mathrm{SSFR} \neq 0$.

${ }^{\mathrm{b}}$ Colors are in the AB system.

addition, we note that BSF ellipticals are more star-forming than S0s, showing on average higher values of SFR and SSFR.

\section{REFERENCES}

Abadi, M. G., Moore, B., \& Bower, R. G. 1999, MNRAS, 308, 947

Abraham, R. G., Valdes, F., Yee, H. K. C., \& van den Bergh, S. 1994, ApJ, 432, 75

Abramson, L. E., Kelson, D. D., Dressler, A., et al. 2014, ApJL, 785, L36

Allen, P. D., Driver, S. P., Graham, A. W., et al. 2006, MNRAS, 371, 2

Bahé, Y. M., McCarthy, I. G., Balogh, M. L., \& Font, A. S. 2013, MNRAS, 430, 3017

Baldry, I. K., Glazebrook, K., Brinkmann, J., et al. 2004, ApJ, 600, 681

Balogh, M., Eke, V., Miller, C., et al. 2004, MNRAS, 348, 1355

Balogh, M. L., Morris, S. L., Yee, H. K. C., Carlberg, R. G., \& Ellingson, E. 1999, ApJ, 527, 54

Balogh, M. L., Navarro, J. F., \& Morris, S. L. 2000a, ApJ, 540, 113

Balogh, M. L., Navarro, J. F., \& Morris, S. L. 2000b, ApJ, 540, 113

Bamford, S. P., Nichol, R. C., Baldry, I. K., et al. 2009, MNRAS, 393, 1324

Bell, E. F., \& de Jong, R. S. 2001, ApJ, 550, 212

Berlind, A. A., Frieman, J., Weinberg, D. H., et al. 2006, ApJS, 167, 1

Blanton, M. R., Eisenstein, D., Hogg, D. W., Schlegel, D. J., \& Brinkmann, J. 2005, ApJ, 629, 143

Blanton, M. R., Hogg, D. W., Bahcall, N. A., et al. 2003, ApJ, 594, 186

Bluck, A. F. L., Mendel, J. T., Ellison, S. L., et al. 2014, MNRAS, 441, 599

Boselli, A., \& Gavazzi, G. 2006, PASP, 118, 517

Brinchmann, J., Charlot, S., White, S. D. M., et al. 2004, MNRAS, 351, 1151

Brough, S., Couch, W. J., Collins, C. A., et al. 2008, MNRAS, 385, L103

Calvi, R., Poggianti, B. M., Fasano, G., \& Vulcani, B. 2012, MNRAS, 419, L14

Calvi, R., Poggianti, B. M., \& Vulcani, B. 2011, MNRAS, 416, 727

Calvi, R., Poggianti, B. M., Vulcani, B., \& Fasano, G. 2013, MNRAS, 432, 3141

Carollo, C. M., Cibinel, A., Lilly, S. J., et al. 2014, arXiv:1402.1172

Christlein, D., \& Zabludoff, A. I. 2005, ApJ, 621, 201

Cid Fernandes, R., Mateus, A., Sodré, L., Stasińska, G., \& Gomes, J. M. 2005, MNRAS, 358, 363

Cimatti, A., Cassata, P., Pozzetti, L., et al. 2008, A\&A, 482, 21

Cole, S., Lacey, C. G., Baugh, C. M., \& Frenk, C. S. 2000, MNRAS, 319, 168

Cowie, L. L., Songaila, A., Hu, E. M., \& Cohen, J. G. 1996, AJ, 112, 839

De Lucia, G., \& Blaizot, J. 2007, MNRAS, 375, 2

De Lucia, G., Weinmann, S., Poggianti, B. M., Aragón-Salamanca, A., \& Zaritsky, D. 2012, MNRAS, 423, 1277

Dekel, A., \& Birnboim, Y. 2006, MNRAS, 368, 2

Dressler, A., Smail, I., Poggianti, B. M., et al. 1999, ApJS, 122, 51

Driver, S. P., Liske, J., Cross, N. J. G., De Propris, R., \& Allen, P. D. 2005, MNRAS, 360, 81

Eke, V. R., Baugh, C. M., Cole, S., et al. 2004, MNRAS, 348, 866

Fasano, G., Vanzella, E., Dressler, A., et al. 2012, MNRAS, 420, 926

Feldmann, R., Carollo, C. M., \& Mayer, L. 2011, ApJ, 736, 88

Ferreras, I., Lisker, T., Pasquali, A., \& Kaviraj, S. 2009, MNRAS, 395, 554

Finn, R. A., Zaritsky, D., McCarthy, D. W., Jr., et al. 2005, ApJ, 630, 206
Font, A. S., Bower, R. G., McCarthy, I. G., et al. 2008, MNRAS, 389, 1619 Forbes, J., Krumholz, M., \& Burkert, A. 2012, ApJ, 754, 48

Fritz, J., Poggianti, B. M., Bettoni, D., et al. 2007, A\&A, 470, 137

Fritz, J., Poggianti, B. M., Cava, A., et al. 2011, A\&A, 526, A45

Gallazzi, A., Bell, E. F., Wolf, C., et al. 2009, ApJ, 690, 1883

Gavazzi, G., Pierini, D., \& Boselli, A. 1996, A\&A, 312, 397

Gehrels, N. 1986, ApJ, 303, 336

Gill, S. P. D., Knebe, A., \& Gibson, B. K. 2005, MNRAS, 356, 1327

Gladders, M. D., Oemler, A., Dressler, A., et al. 2013, ApJ, 770, 64

Gunn, J. E., \& Gott, J. R., III 1972, ApJ, 176, 1

Hashimoto, Y., \& Oemler, A., Jr. 1999, ApJ, 510, 609

Huchra, J. P., \& Geller, M. J. 1982, ApJ, 257, 423

Kannappan, S. J., Guie, J. M., \& Baker, A. J. 2009, AJ, 138, 579

Kauffmann, G., Heckman, T. M., White, S. D. M., et al. 2003, MNRAS, 341,54

Kauffmann, G., White, S. D. M., Heckman, T. M., et al. 2004, MNRAS, 353,713

Kawata, D., \& Mulchaey, J. S. 2008, ApJL, 672, L103

Knobel, C., Lilly, S. J., Iovino, A., et al. 2009, ApJ, 697, 1842

Kovač, K., Lilly, S. J., Knobel, C., et al. 2010, ApJ, 718, 86

Kroupa, P. 2001, MNRAS, 322, 231

Lang, P., Wuyts, S., Somerville, R. S., et al. 2014, ApJ, 788, 11

Larson, R. B., Tinsley, B. M., \& Caldwell, C. N. 1980, ApJ, 237, 692

Lewis, I., Balogh, M., De Propris, R., et al. 2002, MNRAS, 334, 673

Lin, L., Patton, D. R., Koo, D. C., et al. 2008, ApJ, 681, 232

Liske, J., Lemon, D. J., Driver, S. P., Cross, N. J. G., \& Couch, W. J. 2003, MNRAS, 344, 307

Masters, K. L., Mosleh, M., Romer, A. K., et al. 2010, MNRAS, 405, 783

McGee, S. L., Balogh, M. L., Bower, R. G., Font, A. S., \& McCarthy, I. G. 2009, MNRAS, 400, 937

McGee, S. L., Balogh, M. L., Wilman, D. J., et al. 2011, MNRAS, 413, 996

Mok, A., Balogh, M. L., McGee, S. L., et al. 2013, MNRAS, 431, 1090

Moore, B., Katz, N., Lake, G., Dressler, A., \& Oemler, A. 1996, Natur, 379,613

Omand, C. M. B., Balogh, M. L., \& Poggianti, B. M. 2014, MNRAS, 440, 843

Park, C., Gott, J., \& Choi, Y. 2007, BAAS, 39, 771

Pasquali, A., Gallazzi, A., Fontanot, F., et al. 2010, MNRAS, 407, 937

Pasquali, A., van den Bosch, F. C., Mo, H. J., Yang, X., \& Somerville, R. 2009, MNRAS, 394, 38

Peng, Y.-j., Lilly, S. J., Kovač, K., et al. 2010, ApJ, 721, 193

Pimbblet, K. A. 2008, PASA, 25, 176

Poggianti, B. M., Calvi, R., Bindoni, D., et al. 2013, ApJ, 762, 77

Rider, P. R. 1960, JASA, 55, 148

Rudnick, G., Rix, H.-W., Franx, M., et al. 2003, ApJ, 599, 847

Salpeter, E. E. 1955, ApJ, 121, 161

Schawinski, K., Lintott, C., Thomas, D., et al. 2009, MNRAS, 396, 818

Schawinski, K., Urry, C. M., Simmons, B. D., et al. 2014, MNRAS, 440, 889

Simard, L., Willmer, C. N. A., Vogt, N. P., et al. 2002, ApJS, 142, 1

Skibba, R. A., Bamford, S. P., Nichol, R. C., et al. 2009, MNRAS, 399, 966

Skibba, R. A., \& Sheth, R. K. 2009, MNRAS, 392, 1080

Skibba, R. A., Sheth, R. K., \& Martino, M. C. 2007, MNRAS, 382, 1940

Skibba, R. A., van den Bosch, F. C., Yang, X., et al. 2011, MNRAS, 410, 417 
Smith, R. J., Lucey, J. R., Price, J., Hudson, M. J., \& Phillipps, S. 2012, MNRAS, 419, 3167

Tanaka, M., Goto, T., Okamura, S., Shimasaku, K., \& Brinkmann, J. 2004, AJ, 128,2677

Taylor, E. N., Franx, M., van Dokkum, P. G., et al. 2009, ApJS, 183, 295

Tojeiro, R., Masters, K. L., Richards, J., et al. 2013, MNRAS, 432, 359

van den Bosch, F. C., Aquino, D., Yang, X., et al. 2008, MNRAS, 387, 79

van der Wel, A., Rix, H.-W., Holden, B. P., Bell, E. F., \& Robaina, A. R. 2009, ApJL, 706, L120

von der Linden, A., Wild, V., Kauffmann, G., White, S. D. M., \& Weinmann, S. 2010, MNRAS, 404, 1231

Vulcani, B., De Lucia, G., Poggianti, B. M., et al. 2014, ApJ, 788, 57

Walker, I. R., Mihos, J. C., \& Hernquist, L. 1996, ApJ, 460, 121

Wang, H., Mo, H. J., \& Jing, Y. P. 2009, MNRAS, 396, 2249
Weinmann, S. M., Kauffmann, G., van den Bosch, F. C., et al. 2009, MNRAS, 394, 1213

Weinmann, S. M., van den Bosch, F. C., \& Pasquali, A. 2011, in The Dependence of Low Redshift Galaxy Properties on Environment, ed. I. Ferreras \& A Pasquali (Berlin: Springer-Verlag), 29

Weinmann, S. M., van den Bosch, F. C., Yang, X., \& Mo, H. J. 2006, MNRAS, 366,2

Wetzel, A. R., Tinker, J. L., Conroy, C., \& Bosch, F. C. v. d. 2014, MNRAS, 439, 2687

Wetzel, A. R., Tinker, J. L., Conroy, C., \& van den Bosch, F. C. 2013, MNRAS, 432, 336

Wheeler, C., Phillips, J. I., Cooper, M. C., Boylan-Kolchin, M., \& Bullock, J. S. 2014, MNRAS, 442, 1396

Wolf, C., Aragón-Salamanca, A., Balogh, M., et al. 2009, MNRAS, 393, 1302 